\title{
A Class of Spectral Element Methods and Its A Priori/A Posteriori Error Estimates for 2nd-Order Elliptic Eigenvalue Problems
}

\author{
Jiayu Han and Yidu Yang \\ School of Mathematics and Computer Science, Guizhou Normal University, Guiyang 550001, China \\ Correspondence should be addressed to Yidu Yang; ydyang@gznu.edu.cn
}

Received 24 May 2013; Accepted 1 September 2013

Academic Editor: Rasajit Bera

Copyright (C) 2013 J. Han and Y. Yang. This is an open access article distributed under the Creative Commons Attribution License, which permits unrestricted use, distribution, and reproduction in any medium, provided the original work is properly cited.

This paper discusses spectral and spectral element methods with Legendre-Gauss-Lobatto nodal basis for general 2nd-order elliptic eigenvalue problems. The special work of this paper is as follows. (1) We prove a priori and a posteriori error estimates for spectral and spectral element methods. (2) We compare between spectral methods, spectral element methods, finite element methods and their derived $p$-version, $h$-version, and $h p$-version methods from accuracy, degree of freedom, and stability and verify that spectral methods and spectral element methods are highly efficient computational methods.

\section{Introduction}

As we know, finite element methods are local numerical methods for partial differential equations and particularly well suitable for problems in complex geometries, whereas spectral methods can provide a superior accuracy, at the expense of domain flexibility. Spectral element methods combine the advantages of the above methods (see [1]). So far, spectral and spectral element methods are widely applied to boundary value problems (see $[1,2]$ ), as well as applied to symmetric eigenvalue problems (see [3]). However, it is still a new subject to apply them to nonsymmetric elliptic eigenvalue problems.

A posteriorii error estimates and highly efficient computational methods for finite elements of eigenvalue problems are the subjects focused on by the academia these years; see [3-16], and among them, for nonsymmetric 2nd-order elliptic eigenvalue problems, $[5,15]$ provide a posteriori error estimates and adaptive algorithms, [9] the function value recovery techniques and $[8,10]$ two-level discretization schemes.

Based on the work mentioned above, this paper shall further apply spectral and spectral element methods to nonsymmetric elliptic eigenvalue problems. This paper will mainly perform the following work.

(1) We prove a priori and a posteriori error estimates of spectral and spectral element methods with Legendre-Gauss-Lobatto nodal basis, respectively, for the general 2nd-order elliptic eigenvalue problems.

(2) We compare between spectral methods, spectral element methods with Legendre-Gauss-Lobatto nodal basis, finite element methods, and their derived $p$ version, $h$-version, and $h p$-version methods from accuracy, degree of freedom, and stability and verify that spectral methods and spectral element methods are highly efficient computational methods for nonsymmetric 2 nd-order elliptic eigenvalue problems.

This paper is organized as follows. Section 2 introduces basic knowledge of second elliptic eigenvalue problems. Sections 3 and 4 are devoted to a priori and a posteriori error estimates of spectral and spectral element methods, respectively. In Section 5, some numerical experiments are performed by the methods mentioned above. 


\section{Preliminaries}

Consider the 2nd-order elliptic boundary value problem

$$
\begin{gathered}
L w=-\nabla \cdot(D \nabla w)+\mathbf{b} \cdot \nabla w+c w=f, \quad \text { in } \Omega, \\
w=0, \quad \text { on } \partial \Omega,
\end{gathered}
$$

where $\Omega \subset R^{d}(d=2,3)$ is a bounded domain, $\mathbf{b}$ and $c$ are a real-valued vector function and a real-valued function, respectively, and $D$ is a positive scalar function with $D(x) \geq$ $D_{0}>0(\forall x \in \Omega)$.

We denote the complex Sobolev spaces with norm $\|\cdot\|_{m}$ by $H^{m}(\Omega), H_{0}^{1}(\Omega)=\left\{v \in H^{1}(\Omega),\left.v\right|_{\partial \Omega}=0\right\}$. Let $(\cdot, \cdot)$ and $\|\cdot\|_{0, \Omega}$ be a inner product and a norm in the complex space $L^{2}(\Omega)$, respectively.

In this paper, $C$ denotes a generic positive constant independent of the polynomial degrees and mesh scales, which may not be the same at different occurrences.

Define the bilinear form $a(\cdot, \cdot)$ as follows:

$$
\begin{aligned}
& a(w, v)=\int_{\Omega} D \nabla w \nabla \bar{v}+(\mathbf{b} \cdot \nabla w) \bar{v} \\
&+c w \bar{v}, \quad \forall w, v \in H_{0}^{1}(\Omega) .
\end{aligned}
$$

We assume that $f \in L^{2}(\Omega), D, \mathbf{b}$, and $c$ are bounded functions on $\Omega$, namely $D, c \in L^{\infty}(\Omega), \mathbf{b} \in\left(L^{\infty}(\Omega)\right)^{d}$. Further more, we assume that $\nabla \cdot \mathbf{b}$ exists and satisfies

$$
-\frac{1}{2} \nabla \cdot \mathbf{b}+c \geq 0, \quad \text { in } \Omega
$$

Under these assumptions, the bilinear form $a(\cdot, \cdot)$ is continuous in $H_{0}^{1}(\Omega)$ and $H_{0}^{1}(\Omega)$-elliptic; that is, there exist two constants $A, B>0$ independent of $w, v$ such that

$$
\begin{gathered}
|a(w, v)| \leq A\|w\|_{1, \Omega}\|v\|_{1, \Omega}, \quad \forall w, v \in H_{0}^{1}(\Omega), \\
\operatorname{Re} a(v, v) \geq B\|v\|_{1, \Omega}^{2}, \quad \forall v \in H_{0}^{1}(\Omega) .
\end{gathered}
$$

The corresponding variational formulation of (1) is given as follows: find $w \in H_{0}^{1}(\Omega)$, such that

$$
a(w, v)=(f, v), \quad \forall v \in H_{0}^{1}(\Omega) .
$$

The adjoint problem of (5) is as follows: find $w^{*} \in H_{0}^{1}(\Omega)$, such that

$$
a\left(v, w^{*}\right)=(v, f), \quad \forall v \in H_{0}^{1}(\Omega) .
$$

As the general 2nd-order elliptic boundary value problems, we assume that the regularity estimates for problem (5) and its adjoint problem (6) hold, respectively. Namely

$$
\begin{array}{cl}
\|w\|_{r_{1}+1, \Omega} \leq C\|f\|_{0, \Omega}, & 0<r_{1} \leq 1, \\
\left\|w^{*}\right\|_{r_{2}+1, \Omega} \leq C\|f\|_{0, \Omega}, & 0<r_{2} \leq 1 .
\end{array}
$$

We assume that $K_{h}=\{\kappa\}$ is a regular rectangle (resp. cuboid) or simplex partition of the domain $\Omega$ and satisfies
$\bar{\Omega}=\bigcup \bar{\kappa}$. We associate with the partition a polynomial degree vector $\mathbf{N}=\left\{N_{\kappa}\right\}$, where $N_{\kappa}$ is the polynomial degree in $\kappa$. Let $h_{\kappa}$ be the diameter of the element $\kappa$, and let $h=\max _{\kappa \in K_{h}} h_{\kappa}$.

We define spectral and spectral element spaces as follows:

$$
\begin{gathered}
S_{N}(\Omega)=\left\{v \in P_{N}(\Omega),\left.v\right|_{\partial \Omega}=0\right\}, \\
S_{N, h}(\Omega)=\left\{v \in C(\bar{\Omega}):\left.v\right|_{\mathcal{\kappa}} \in P_{N_{\kappa}}(\kappa), \forall \kappa \in K_{h},\left.v\right|_{\partial \Omega}=0\right\},
\end{gathered}
$$

where $P_{N}(\Omega)$ and $P_{N_{\kappa}}(\kappa)$ are polynomial spaces of degree $N$ (resp. degree $N$ in every direction) in $\Omega$ and degree $N_{\kappa}$ (resp. degree $N_{\kappa}$ in every direction) in the element $\kappa$, respectively.

The spectral approximation of (5) is as follows: find $w_{N} \in$ $S_{N}(\Omega)$, such that

$$
a\left(w_{N}, v\right)=(f, v), \quad \forall v \in S_{N}(\Omega)
$$

The spectral element approximation of (5) is as follows: find $w_{N, h} \in S_{N, h}(\Omega)$, such that

$$
a\left(w_{N, h}, v\right)=(f, v), \quad \forall v \in S_{N, h}(\Omega)
$$

We assume that $f \in L^{2}(\Omega)$ and derive from Lax-Milgram theorem that the variational formations (5), (6), (10), and (11) have a unique solution, respectively.

Define the interpolation operators

$$
\begin{gathered}
I_{N_{\kappa}, h_{\kappa}}: C(\kappa) \longrightarrow P_{N_{\kappa}}(\kappa), \\
I_{N}: C(\Omega) \longrightarrow S_{N}(\Omega),
\end{gathered}
$$

as the interpolations in the element $\kappa$ and the domain $\Omega$, respectively, with the tensorial Legendre-Gauss-Lobatto (LGL) interpolation nodes.

Define the interpolation operator

$$
I_{N, h}: C(\Omega) \longrightarrow S_{N, h}(\Omega), \quad \text { satisfying }\left.I_{N, h}\right|_{\kappa}=I_{N_{\kappa}, h_{\kappa}}
$$

We quote from [2] (see (5.8.27) therein) the interpolation estimates for spectral and spectral element methods with LGL Nodal-basis as follows.

For all $v \in H^{s_{\kappa}}(\kappa), s_{\kappa} \geq(d+1) / 2$,

$$
\begin{gathered}
\left\|v-I_{N_{\kappa}, h_{\kappa}} v\right\|_{1, \kappa} \leq C\left(s_{\kappa}\right) h_{\kappa}^{\min \left(N_{\kappa}+1, s_{\kappa}\right)-1} N_{\kappa}^{-s_{\kappa}+1}\|v\|_{s_{\kappa}, \kappa}, \\
\left\|v-I_{N_{\kappa}, h_{\kappa}} v\right\|_{0, \kappa} \leq C\left(s_{\kappa}\right) h_{\kappa}^{\min \left(N_{\kappa}+1, s_{\kappa}\right)} N_{\kappa}^{-s_{\kappa}}\|v\|_{s_{\kappa}, \kappa} .
\end{gathered}
$$

For all $v \in H^{s}(\Omega), s \geq(d+1) / 2$,

$$
\begin{gathered}
\left\|v-I_{N} v\right\|_{1, \Omega} \leq C(s) N^{-s+1}\|v\|_{s, \Omega}, \\
\left\|v-I_{N} v\right\|_{0, \Omega} \leq C(s) N^{-s}\|v\|_{s, \Omega} .
\end{gathered}
$$

We assume that the solution of boundary value problem (5) $w \in H_{0}^{1}(\Omega) \cap H^{m}(\Omega)(m>1)$, that $w_{N}$ and $w_{N, h}$ are the 
solutions of (10) and (11), respectively; then we derive from Céa lemma and the interpolation estimates that

$$
\begin{aligned}
\left\|w_{N}-w\right\|_{1, \Omega} \leq & C(m) N^{-m+1}\|w\|_{m, \Omega}, \\
\left\|w_{N, h}-w\right\|_{1, \Omega} \leq & \left\{\sum_{\kappa} C\left(s_{\kappa}\right) h_{\kappa}^{2\left\{\min \left(N_{\kappa}+1, s_{\kappa}\right)-1\right\}}\right. \\
& \left.\times N_{\kappa}^{2\left(-s_{\kappa}+1\right)}\|w\|_{s_{\kappa}, \kappa}^{2}\right\}^{1 / 2},
\end{aligned}
$$

where $s_{\kappa} \geq(d+1) / 2, \forall \kappa \in K_{h}$.

Particularly, if $N_{\kappa}=N, \forall \kappa \in K_{h}$, then we have

$$
\begin{aligned}
\left\|w_{N, h}-w\right\|_{1, \Omega} \leq & C(m) h^{\min (N+1, m)-1} \\
& \times N^{1-m}\|w\|_{m, \Omega} .
\end{aligned}
$$

Note that (18) is also suited to spectral methods with modal basis (see $[1,2])$.

Using Aubin-Nitsche technique, we deduce from the regularity estimate (8) and the estimates (18)-(20) the priori estimates of boundary value problem (5) for spectral and spectral element methods; that is,

$$
\begin{gathered}
\left\|w_{N}-w\right\|_{0, \Omega} \leq C N^{-m-r_{2}+1}\|w\|_{m, \Omega}, \\
\left\|w_{N, h}-w\right\|_{0, \Omega} \leq C N^{-m-r_{2}+1} h^{r_{2}+\min (N+1, m)-1}\|w\|_{m, \Omega} .
\end{gathered}
$$

\section{Spectral and Spectral-Element Approximations and Error Estimates for Eigenvalue Problems}

3.1. Spectral and Spectral-Element Approximations for Eigenvalue Problems. Consider the following eigenvalue problem corresponding to the boundary value problem (1):

$$
\begin{gathered}
L u=\lambda u, \quad \text { in } \Omega, \\
u=0, \quad \text { on } \partial \Omega .
\end{gathered}
$$

The variational formation of (23) is given by the following: find $\lambda \in \mathbb{C}, 0 \neq u \in H_{0}^{1}(\Omega)$, such that

$$
a(u, v)=\lambda(u, v), \quad \forall v \in H_{0}^{1}(\Omega)
$$

The spectral approximation scheme of (24) is given by the following: find $\lambda_{N} \in \mathbb{C}, 0 \neq u_{N} \in S_{N}(\Omega)$, such that

$$
a\left(u_{N}, v_{N}\right)=\lambda_{N}\left(u_{N}, v_{N}\right), \quad \forall v_{N} \in S_{N}(\Omega) \text {. }
$$

The spectral element approximation scheme of (24) is given by the following: find $\lambda_{N, h} \in \mathbb{C}, 0 \neq u_{N, h} \in S_{N, h}(\Omega)$, such that

$$
a\left(u_{N, h}, v_{N, h}\right)=\lambda_{N, h}\left(u_{N, h}, v_{N, h}\right), \quad \forall v_{N, h} \in S_{N, h}(\Omega),
$$

Define the solution operators $T: L^{2}(\Omega) \rightarrow H_{0}^{1}(\Omega), T_{N}:$ $L^{2}(\Omega) \rightarrow S_{N}(\Omega)$, and $T_{N, h}: L^{2}(\Omega) \rightarrow S_{N, h}(\Omega)$ by

$$
\begin{array}{r}
a(T f, v)=(f, v), \quad \forall f \in L^{2}(\Omega), \quad \forall v \in H_{0}^{1}(\Omega), \\
a\left(T_{N} f, v_{N}\right)=\left(f, v_{N}\right), \quad \forall f \in L^{2}(\Omega), \\
\forall v_{N} \in S_{N}(\Omega), \\
a\left(T_{N, h} f, v_{N, h}\right)=\left(f, v_{N, h}\right), \quad \forall f \in L^{2}(\Omega), \\
\forall v_{N, h} \in S_{N, h}(\Omega) .
\end{array}
$$

Obviously (see [17]), the equivalent operator forms for (24) and (26) are the following.

Find $\lambda \in \mathbb{C}, 0 \neq u \in H_{0}^{1}(\Omega)$, such that

$$
T u=\lambda^{-1} u \text {. }
$$

Find $\lambda_{N, h} \in \mathbb{C}, 0 \neq u_{N, h} \in S_{N, h}(\Omega)$, such that

$$
T_{N, h} u_{N, h}=\lambda_{N, h}^{-1} u_{N, h}
$$

The adjoint problem of the eigenvalue problem (23) is

$$
\begin{gathered}
L^{*} u^{*}=\lambda^{*} u^{*}, \quad \text { in } \Omega, \\
u^{*}=0, \quad \text { on } \partial \Omega,
\end{gathered}
$$

where $L^{*} u^{*}=-\nabla \cdot\left(D \nabla u^{*}\right)-\mathbf{b} \cdot \nabla u^{*}+(c-\nabla \cdot \mathbf{b}) u^{*}$.

The variational formation of (30) is given by the following: find $\lambda^{*} \in \mathbb{C}, 0 \neq u^{*} \in H_{0}^{1}(\Omega)$, such that

$$
a^{*}\left(u^{*}, v\right):=\overline{a\left(v, u^{*}\right)}=\lambda^{*}\left(u^{*}, v\right), \quad \forall v \in H_{0}^{1}(\Omega) .
$$

The spectral element approximation scheme of (31) is given by the following: find $\lambda_{N, h}^{*} \in \mathbb{C}, 0 \neq u_{N, h}^{*} \in S_{N, h}(\Omega)$, such that

$$
a^{*}\left(u_{N, h}^{*}, v_{N, h}\right)=\lambda_{N, h}^{*}\left(u_{N, h}^{*}, v_{N, h}\right), \quad \forall v_{N, h} \in S_{N, h}(\Omega) .
$$

We can likewise define the equivalent operator forms for the eigenvalue problems (31) and (32) as

$$
\begin{gathered}
T^{*} u^{*}=\lambda^{*-1} u^{*}, \\
T_{N, h}^{*} u_{N, h}^{*}=\lambda_{N, h}^{*-1} u_{N, h}^{*} .
\end{gathered}
$$

Let $\lambda$ be an eigenvalue of (23). There exists a smallest integer $\mu$, called the ascent of $\lambda$, such that $\operatorname{ker}\left(\left(\lambda^{-1}-T\right)^{\mu}\right)=$ $\operatorname{ker}\left(\left(\lambda^{-1}-T\right)^{\mu+1}\right) \cdot q=\operatorname{dim} \operatorname{ker}\left(\left(\lambda^{-1}-T\right)^{\mu}\right)$ is called the algebraic multiplicity of $\lambda$. The functions in $\operatorname{ker}\left(\left(\lambda^{-1}-T\right)^{\mu}\right)$ are called generalized eigenfunctions of $T$ corresponding to $\lambda$. Likewise the ascent, algebraic multiplicity and generalized eigenfunctions of $\lambda_{N, h}, \lambda^{*}$ and $\lambda_{N, h}^{*}$ can be defined.

Let $\lambda$ be an eigenvalue of (23) with the algebraic multiplicity $q$ and the ascent $\mu$. Assume $\left\|T_{N, h}-T\right\|_{1, \Omega} \rightarrow 0(N \rightarrow \infty$, $h \rightarrow 0)$; then there are eigenvalues $\lambda_{j, N, h}(j=1,2, \ldots, q)$ of (26) which converge to $\lambda$. Let $M(\lambda)$ be the space spanned by all generalized eigenfunctions corresponding to $\lambda$ of $T$, 
and let $M_{N, h}(\lambda)$ be the space spanned by all generalized eigenfunctions corresponding to all eigenvalues of $T_{N, h}$ that converge to $\lambda$.

In view of adjoint problems (31) and (32), the definitions of $M^{*}\left(\lambda^{*}\right)$ and $M_{N, h}^{*}\left(\lambda^{*}\right)$ are analogous to $M(\lambda)$ and $M_{N, h}(\lambda)$. Let $\widehat{M}(\lambda)=\left\{v \in M(\lambda):\|v\|_{1, \Omega}=1\right\}$, and let $\widehat{M^{*}}\left(\lambda^{*}\right)=\{v \in$ $\left.M^{*}\left(\lambda^{*}\right):\|v\|_{1, \Omega}=1\right\}$.

Note that when $\mathbf{b}=0$, both (24) and (26) are symmetric. Thus, the ascent $\mu=1$ of $\lambda$, and the ascent $l=1$ of $\lambda_{N, h}$.

3.2. A Priori Error Estimates. We will analyze a prior error estimates for spectral element methods which are suitable for spectral methods with mesh fineness $h$ not considered.

Assume that $R$ and $U$ are two closed subspace in $H_{0}^{1}(\Omega)$.

Denote

$$
\begin{gathered}
\delta(R, U)=\sup _{\substack{v \in R \\
\|v\|_{1, \Omega}=1}} \operatorname{dist}(v, U), \\
\theta(R, U)=\max (\delta(R, U), \delta(U, R)) .
\end{gathered}
$$

We say that $\theta(R, U)$ is the gap between $R$ and $U$.

Denote

$$
\begin{gathered}
\varepsilon_{N, h}=\varepsilon_{N, h}(\lambda)=\sup _{u \in \widehat{M}(\lambda)^{v \in S_{N, h}}} \inf _{(\Omega)}\|u-v\|_{1, \Omega}, \\
\varepsilon_{N, h}^{*}=\varepsilon_{N, h}^{*}\left(\lambda^{*}\right)=\sup _{u \in \widehat{M}^{*}\left(\lambda^{*}\right)^{*}} \inf _{v \in S_{N, h}(\Omega)}\|u-v\|_{1, \Omega} .
\end{gathered}
$$

We give the following four lemmas from Theorem 8.1-8.4 in [17], which are applications to spectral element methods.

Lemma 1. Assume $\left\|T_{N, h}-T\right\|_{1, \Omega} \rightarrow 0(N \rightarrow \infty, h \rightarrow 0)$. For small enough $h$ and big enough $N$, there holds

$$
\theta\left(M(\lambda), M_{N, h}(\lambda)\right) \leq C \varepsilon_{N, h} .
$$

Lemma 2. Assume $\left\|T_{N, h}-T\right\|_{1, \Omega} \rightarrow 0(N \rightarrow \infty, h \rightarrow 0)$; then

$$
\left|\lambda^{-1}-\frac{1}{q} \sum_{j=1}^{q} \lambda_{j, N, h}^{-1}\right| \leq C \varepsilon_{N, h} \varepsilon_{N, h}^{*} .
$$

Lemma 3. Assume that $\left\|T_{N, h}-T\right\|_{1, \Omega} \rightarrow 0(N \rightarrow \infty, h \rightarrow$ $0)$; then there holds

$$
\left|\lambda-\lambda_{j, N, h}\right| \leq C\left(\varepsilon_{N, h} \varepsilon_{N, h}^{*}\right)^{1 / \mu} \quad(j=1,2, \ldots, q) .
$$

Since $\operatorname{ker}\left(\left(\lambda^{-1}-T\right)^{l}\right)(l \geq 1)$ is a finite dimensional space, there exists a direct-sum decomposition $H_{0}^{1}(\Omega)=\operatorname{ker}\left(\left(\lambda^{-1}-T\right)^{l}\right) \oplus$ $M_{l}$. We define the operator $E_{l}$ as a projection along $M_{l}$ from $H_{0}^{1}(\Omega)$ to $\operatorname{ker}\left(\left(\lambda^{-1}-T\right)^{l}\right)$.

Lemma 4. Assume $\left\|T_{N, h}-T\right\|_{1, \Omega} \rightarrow 0(N \rightarrow \infty, h \rightarrow 0)$. Let $\lambda_{N, h}$ be an eigenvalue of $T_{N, h}$ and $\lim _{N \rightarrow \infty, h \rightarrow 0} \lambda_{N, h}=\lambda$. $u_{N, h}$ satisfies $\left(\lambda_{N, h}^{-1}-T_{N, h}\right)^{k} u_{N, h}=0$ and $\left\|u_{N, h}\right\|_{1, \Omega}=1$, where $k \leq \mu$ is a positive integer. Then, for every integer $l \in[k, \mu]$, there holds

$$
\left\|u_{N, h}-E_{l} u_{N, h}\right\|_{1, \Omega} \leq C \varepsilon_{N, h}^{(l-k+1) / \mu}
$$

We assume that in this section, for the sake of simplicity, $N_{\kappa}=N, \forall \kappa \in K_{h}$.

Theorem 5. If $M(\lambda) \subset H^{t_{1}}(\Omega)$ and $M^{*}\left(\lambda^{*}\right) \subset H^{t_{2}}(\Omega)$, then there holds the following error estimates:

$$
\begin{aligned}
& \left|\frac{1}{q} \sum_{j=1}^{q} \lambda_{j, N, h}-\lambda\right| \\
& \leq C\left(\frac{h^{\tau_{1}+\tau_{2}-2}}{N^{t_{1}+t_{2}-2}}\right) \sup _{u \in \widehat{M}(\lambda)}\|u\|_{t_{1}, \Omega} \sup _{v \in \widehat{M^{*}}\left(\lambda^{*}\right)}\|v\|_{t_{2}, \Omega}, \\
& \left|\lambda_{j, N, h}-\lambda\right| \\
& \leq C\left(\left(\frac{h^{\tau_{1}+\tau_{2}-2}}{N^{t_{1}+t_{2}-2}}\right) \sup _{u \in \widehat{M}(\lambda)}\|u\|_{t_{1}, \Omega} \underset{v \in \widehat{M^{*}\left(\lambda^{*}\right)}}{\sup _{t_{2}, \Omega}}\right)^{1 / \mu} \\
& \theta\left(M(\lambda), M_{N, h}(\lambda)\right) \leq C \frac{h^{\tau_{1}-1}}{N^{t_{1}-1}} \sup _{u \in \widehat{M}(\lambda)}\|u\|_{t_{1}, \Omega} .
\end{aligned}
$$

Let $\left\|u_{N, h}\right\|_{1, \Omega}=1$, and let $\left(\lambda_{N, h}^{-1}-T_{N, h}\right)^{l_{1}} u_{N, h}=0$, for some $l_{1} \leq \mu$. Then, for every integer $l_{2}\left(l_{1} \leq l_{2} \leq \mu\right)$, there exists a function $u^{\prime}$, such that $\left(\lambda^{-1}-T\right)^{l_{2}} u^{\prime}=0$ and

$$
\left\|u_{N, h}-u^{\prime}\right\|_{1, \Omega} \leq C\left(\left(\frac{h^{\tau_{1}-1}}{N^{t_{1}-1}}\right) \sup _{u \in \widehat{M}(\lambda)}\|u\|_{t_{1}, \Omega}\right)^{\left(l_{2}-l_{1}+1\right) / \mu},
$$

where $\tau_{1}=\min \left(N+1, t_{1}\right), \tau_{2}=\min \left(N+1, t_{2}\right)$.

Proof. We derive from the error estimate (20) that

$$
\begin{aligned}
& \left\|T_{N, h}-T\right\|_{1, \Omega} \\
& \quad=\sup _{f \in H_{0}^{1}(\Omega)} \frac{\left\|\left(T-T_{N, h}\right) f\right\|_{1, \Omega}}{\|f\|_{1, \Omega}} \\
& \quad \leq C\left(1+r_{1}\right) h^{r_{1}} N^{-r_{1}} \longrightarrow 0 \quad(N \longrightarrow \infty, h \longrightarrow 0) .
\end{aligned}
$$

By (14),

$$
\begin{aligned}
\varepsilon_{N, h}=\varepsilon_{N, h}(\lambda) & =\sup _{u \in \widehat{M}(\lambda)^{v \in S_{N, h}(\Omega)}} \inf _{1}\|u-v\|_{1, \Omega} \\
& \leq C\left(\frac{h^{\tau_{1}-1}}{N^{t_{1}-1}}\right) \sup _{u \in \widehat{M}(\lambda)}\|u\|_{t_{1}, \Omega} .
\end{aligned}
$$

Analogically,

$$
\varepsilon_{N, h}^{*} \leq C\left(\frac{h^{\tau_{2}-1}}{N^{t_{2}-1}}\right) \sup _{u \in \widehat{M^{*}\left(\lambda^{*}\right)}}\|u\|_{t_{2}, \Omega} .
$$


Plugging the two inequalities above into (36), (38), and (39) yields (42), (41), and (43), respectively. We find from (37) that

$$
\begin{aligned}
\left|\frac{1}{q} \sum_{j=1}^{q} \lambda_{j, N, h}-\lambda\right| & =\left|\frac{1}{q} \sum_{j=1}^{q} \frac{\lambda_{j, N, h}^{-1}-\lambda^{-1}}{\lambda^{-1} \lambda_{j, N, h}^{-1}}\right| \\
& \leq C\left|\frac{1}{q} \sum_{j=1}^{q} \lambda_{j, N, h}^{-1}-\lambda^{-1}\right| \leq C \varepsilon_{N, h} \varepsilon_{N, h}^{*},
\end{aligned}
$$

combining with (45) and (46) yields (40).

Supposing that $\left\|T_{N, h}-T\right\|_{0, \Omega} \rightarrow 0(N \rightarrow \infty, h \rightarrow 0)$, $\rho(T)$ is a regular set of $T$, and $\Gamma \subset \rho(T)$ is a closed Jordan curve enclosing $\lambda^{-1}$.

Denote

$$
\begin{gathered}
R(z)=(T-z)^{-1}, \\
R\left(T_{N, h}, z\right)=\left(T_{N, h}-z\right)^{-1} .
\end{gathered}
$$

Define the spectral projection operators

$$
\begin{gathered}
E=\frac{-1}{2 i \pi} \int_{\Gamma} R(T, z) \mathrm{d} z: H_{0}^{1}(\Omega) \longrightarrow M(\lambda), \\
E_{N, h}=\frac{-1}{2 i \pi} \int_{\Gamma} R\left(T_{N, h}, z\right) \mathrm{d} z: H_{0}^{1}(\Omega) \longrightarrow M_{N, h}(\lambda) .
\end{gathered}
$$

We give the following lemma by referring to $[18,19]$ (see proposition 5.3 in [18] and theorem 1.3.2 in [19]).

Lemma 6. If $\left\|T_{N, h}-T\right\|_{0, \Omega} \rightarrow 0(N \rightarrow \infty, h \rightarrow 0)$, then there holds that $E_{N, h} \rightarrow E(p), R\left(T_{N, h}, z\right)$ is uniformly bounded with $N$ and $h$, and

$$
\begin{gathered}
\left\|\left(E_{N, h}-E\right) v\right\|_{0, \Omega} \leq \max _{z \in \Gamma}\left\|\left(T-T_{N, h}\right) R(z) v\right\|_{0, \Omega}, \\
\forall v \in H_{0}^{1}(\Omega), \\
\left\|\left(E_{N, h}-E\right) v\right\|_{0, \Omega} \leq \max _{z \in \Gamma}\left\|\left(T-T_{N, h}\right) R\left(T_{N, h}, z\right) v\right\|_{0, \Omega},
\end{gathered}
$$$$
\forall v \in H_{0}^{1}(\Omega)
$$

Theorem 7. Under the assumptions of Theorem 5, further assume that the ascent of $\lambda$ is $\mu=1$. Let $\left(\lambda_{N, h}, u_{N, h}\right)$ be an eigenpair of (26) with $\left\|u_{N, h}\right\|_{0, \Omega}=1$; then there exists an eigenpair $(\lambda, u)$ of $(24)$, such that

$$
\begin{gathered}
\left\|u_{N, h}-u\right\|_{1, \Omega} \leq \frac{C h^{\tau_{1}-1}\|u\|_{t_{1}, \Omega}}{N^{t_{1}-1}}, \\
\left\|u_{N, h}-u\right\|_{0, \Omega} \leq \frac{C h^{r_{2}+\tau_{1}-1}\|u\|_{t_{1}, \Omega}}{N^{r_{2}+t_{1}-1}}, \\
\leq \lambda_{N, h}-\lambda \mid \\
\leq C\left(\left(\frac{h^{\tau_{1}+\tau_{2}-2}}{N^{t_{1}+t_{2}-2}}\right) \sup _{u \in \widehat{M}(\lambda)}\|u\|_{t_{1}, \Omega} \sup _{v \in \widehat{M^{*}}\left(\lambda^{*}\right)}\|v\|_{t_{2}, \Omega}\right),
\end{gathered}
$$

where $\tau_{1}=\min \left(N+1, t_{1}\right)$ and $\tau_{2}=\min \left(N+1, t_{2}\right)$.
Let $(\lambda, u)$ be an eigenpair of (24). If $\lambda_{N, h}$ is an eigenvalue of (26) convergence to $\lambda$, then there exists $u_{N, h} \in \operatorname{ker}\left(\lambda_{N, h}^{-1}-T_{N, h}\right)$, such that (51)-(53) hold.

Proof. We deduce (53) immediately from (41). We derive from (22) and (7) that

$$
\left\|T f-T_{N, h} f\right\|_{0, \Omega} \leq C N^{-r_{1}-r_{2}} h^{r_{1}+r_{2}}\|f\|_{0, \Omega}
$$

thus, $\left\|T-T_{N, h}\right\|_{0, \Omega} \rightarrow 0(N \rightarrow \infty, h \rightarrow 0)$. Taking $u=$ $E u_{N, h}$ and by virtue of $R\left(T_{N, h}, z\right) u_{N, h}=\left(\lambda_{N, h}^{-1}-z\right)^{-1} u_{N, h}$, Lemma 6 and (22), we have

$$
\begin{aligned}
\left\|u-u_{N, h}\right\|_{0, \Omega}= & \left\|E u_{N, h}-E_{N, h} u_{N, h}\right\|_{0, \Omega} \\
\leq & C\left\|\left(T-T_{N, h}\right) u_{N, h}\right\|_{0, \Omega} \\
\leq & C\left(\left\|\left(T-T_{N, h}\right) u\right\|_{0, \Omega}\right. \\
& \left.+\left\|\left(T-T_{N, h}\right)\left(u_{N, h}-u\right)\right\|_{0, \Omega}\right),
\end{aligned}
$$

from which follows

$$
\begin{aligned}
\left\|u-u_{N, h}\right\|_{0, \Omega} & \leq C\left\|\left(T-T_{N, h}\right) u\right\|_{0, \Omega} \\
& \leq \frac{C h^{r_{2}+\tau_{1}-1}\|u\|_{t_{1}, \Omega}}{N^{r_{2}+t_{1}-1}}
\end{aligned}
$$

which is (52). By direct calculation, we have

$$
\begin{aligned}
\| u & -u_{N, h} \|_{1, \Omega} \\
& =\left\|\lambda T u-\lambda_{h} T_{N, h} u_{N, h}\right\|_{1, \Omega} \\
& \leq\left\|\lambda T u-\lambda T_{N, h} u\right\|_{1, \Omega}+\left\|\lambda T_{N, h} u-\lambda_{h} T_{N, h} u_{N, h}\right\|_{1, \Omega} \\
& \leq\left\|\left(T-T_{N, h}\right)(\lambda u)\right\|_{1, \Omega}+C\left\|\lambda u-\lambda_{N, h} u_{N, h}\right\|_{0, \Omega} .
\end{aligned}
$$

Plugging (20), (52), and (53) into (57) yields (51).

If $(\lambda, u)$ is an eigenpair of (24), let $u_{N, h}=E_{N, h} u$; by the same argument we can prove (51) and (52).

\section{A Posteriori Error Estimates}

Based on [20], we will discuss a posteriori error estimates. We further assume that $\Omega \subset R^{2}$, the partition $K_{h}$ is $\gamma$-shape regular, and the polynomial degree of neighboring elements are comparable; that is, there exists $\gamma>0$, such that for all $\kappa, \kappa^{\prime} \in K_{h}, \bar{\kappa} \cap \overline{\kappa^{\prime}} \neq \emptyset$,

$$
\begin{gathered}
\gamma^{-1} h_{\kappa} \leq h_{\kappa^{\prime}} \leq \gamma h_{\kappa}, \\
\gamma^{-1}\left(N_{\kappa}+1\right) \leq N_{\mathcal{\kappa}^{\prime}}+1 \leq \gamma\left(N_{\kappa}+1\right) .
\end{gathered}
$$

We refer to the $h p$-clément interpolation estimates given by $[20,21]$ (see theorems 2.2 and 2.3 , respectively), which generalize the well-known clément type interpolation operators studied in [22] and [23] to the hp context.

Lemma 8. Assume that the partition $K_{h}$ is $\gamma$-shape regular and the polynomial distribution $\mathbf{N}$ is comparable. Then there 
exists a positive constant $C=C(\gamma)$ and the clement operator $I: H_{0}^{1}(\Omega) \rightarrow S_{N, h}(\Omega)$, such that

$$
\begin{gathered}
\|v-I v\|_{0, \kappa} \leq C \frac{h_{\kappa}}{N_{\kappa}}\|\nabla v\|_{0, \omega_{\kappa}}, \\
\|v-I v\|_{0, e} \leq C \sqrt{\frac{h_{e}}{N_{e}}}\|\nabla v\|_{0, \omega_{e}},
\end{gathered}
$$

where $h_{e}$ is the length of the edge e and $N_{e}=\max \left(N_{\kappa_{1}}, N_{\kappa_{2}}\right)$, where $\kappa_{1}, \kappa_{2}$ are elements sharing the edge e and $\omega_{\kappa}, \omega_{e}$ are patches covering $\kappa$ and $e$ with a few layers, respectively.

Define interval $\widehat{I}=(0,1)$ and weight function $\Phi_{\widehat{I}}(x):=$ $x(1-x)$. Denote the reference square and triangle element by $\widehat{\kappa}=(0,1)^{2}$ and $\widehat{\kappa}=\{(x, y) \mid 0<x<1,0<y<\sqrt{3}(1 / 2-\mid 1 / 2-$ $x \mid)\}$, respectively. Define weight function $\Phi_{\widehat{\kappa}}(x):=\operatorname{dist}(x, \partial \widehat{\kappa})$.

The following three lemmas are given by [20]. Lemmas 9-10 provide the polynomial inverse estimates in standard interval and element, while Lemma 11 provides a result for the extension from an edge to the element.

Lemma 9. Let $-1<\alpha<\beta, \sigma \in[0,1]$. Then there exists $C=$ $C(\alpha, \beta)$, such that for all $N \in \mathbb{N}$ and all univariate polynomials $\pi_{N}$ of degree $N$,

$$
\begin{aligned}
& \int_{\widehat{I}} \Phi_{\widehat{I}}^{\alpha}(x)\left|\pi_{N}(x)\right|^{2} d x \\
& \quad \leq C N^{2(\beta-\alpha)} \int_{\widehat{I}} \Phi_{\widehat{I}}^{\beta}\left|\pi_{N}(x)\right|^{2} d x .
\end{aligned}
$$

Lemma 10. Let $-1<\alpha<\beta, \sigma \in[0,1]$. Then there exist $C_{1}=C(\alpha, \beta), C_{2}=C_{\sigma}>0$, such that for all $N \in \mathbb{N}$ and all polynomials $\pi_{N}$ of degree bi-N,

$$
\begin{aligned}
& \int_{\widehat{\mathcal{K}}} \Phi_{\widehat{\kappa}}^{\alpha}\left|\pi_{N}\right|^{2} d x d y \\
& \leq C_{1} N^{2(\beta-\alpha)} \int_{\widehat{\mathcal{\kappa}}} \Phi_{\widehat{\kappa}}^{\beta}\left|\pi_{N}\right|^{2} d x d y, \\
& \int_{\widehat{\mathcal{K}}} \Phi_{\widehat{\kappa}}^{2 \sigma}\left|\nabla \pi_{N}\right|^{2} d x d y \\
& \leq C_{2} N^{2(2-\sigma)} \int_{\widehat{\mathcal{K}}} \Phi_{\widehat{\kappa}}^{\sigma}\left|\pi_{N}\right|^{2} d x d y .
\end{aligned}
$$

Lemma 11. Let $\alpha \in(1 / 2,1] . \widehat{e}:=(0,1) \times\{0\}, \Phi_{\widehat{e}}:=x(1-x)$; then there exists $C_{\alpha}>0$ such that for all $N \in \mathbb{N}, \varepsilon \in(0,1]$, and all univariate polynomials $\pi$ of degree $N$, there exists an extension $v_{\widehat{e}} \in H^{1}(\widehat{\kappa})$ and holds

$$
\begin{gathered}
\left.v_{\widehat{e}}\right|_{\widehat{e}}=\pi \cdot \Phi_{\widehat{e}}^{\alpha},\left.\quad v_{\widehat{e}}\right|_{\partial \widehat{\kappa} \mid \widehat{e}}=0, \\
\left\|v_{\widehat{e}}\right\|_{0, \widehat{\kappa}}^{2} \leq C_{\alpha} \varepsilon\left\|\pi \Phi_{\widehat{e}}^{\alpha / 2}\right\|_{0, \widehat{e}}^{2} \\
\left\|\nabla v_{\widehat{e}}\right\|_{0, \widehat{\kappa}}^{2} \leq C_{\alpha}\left(\varepsilon N^{2(2-\alpha)}+\varepsilon^{-1}\right)\left\|\pi \Phi_{\widehat{e}}^{\alpha / 2}\right\|_{0, \widehat{e}}^{2}
\end{gathered}
$$

It is easy to know that the three lemmas above hold for complex-valued polynomials.
Let $D_{\kappa}, \mathbf{b}_{\kappa}$, and $c_{\kappa}$ be the interpolations of $D, \mathbf{b}$, and $c$ in $\kappa$ with the polynomial degree $N_{\kappa}$ (resp. degree $N_{\kappa}$ in every direction), respectively, or the $L^{2}(\kappa)$-projection on the space of polynomials with degree $N_{\kappa}$. For convenient argument, here and hereafter we assume that $(\lambda, u)$ and $\left(\lambda^{*}=\bar{\lambda}, u^{*}\right)$ are the eigenpairs of the eigenvalue problem (24) and its adjoint problem (31), respectively. $\left(\lambda_{N, h}, u_{N, h}\right)$ and $\left(\lambda_{N, h}^{*}=\right.$ $\left.\overline{\lambda_{N, h}}, u_{N, h}^{*}\right)$ are the solutions of the corresponding spectral element approximations (26) and (32), respectively.

Denote

$$
\begin{aligned}
L_{\kappa} u_{N, h}:= & -\nabla \cdot\left(D_{\kappa} \nabla u_{N, h}\right) \\
& +\mathbf{b}_{\kappa} \cdot \nabla u_{N, h}+c_{\kappa} u_{N, h}, \\
L_{\kappa}^{*} u_{N, h}^{*}:= & -\nabla \cdot\left(D_{\kappa} \nabla u_{N, h}^{*}\right) \\
& -\mathbf{b}_{\kappa} \cdot \nabla u_{N, h}^{*}+\left(c_{\kappa}-\nabla \cdot \mathbf{b}_{\kappa}\right) u_{N, h}^{*} .
\end{aligned}
$$

Define the local error indicators

$$
\begin{aligned}
& \eta_{\alpha ; \kappa}^{2}:=\eta_{\alpha ; B_{\kappa}}^{2}+\eta_{\alpha ; E_{\kappa}}^{2}, \\
& \eta_{\alpha ; \kappa}^{* 2}:=\eta_{\alpha ; B_{\kappa}}^{* 2}+\eta_{\alpha ; E_{\kappa}}^{* 2} .
\end{aligned}
$$

Their first terms $\eta_{\alpha ; B_{\kappa}}^{2}, \eta_{\alpha ; B_{\kappa}}^{* 2}$ are the weighted element internal residuals given by

$$
\begin{aligned}
& \eta_{\alpha ; B_{\kappa}}^{2}:=\frac{h_{\kappa}^{2}}{N_{\kappa}^{2}}\left\|\left(-L_{\kappa} u_{N, h}+\lambda_{N, h} u_{N, h}\right) \Phi_{\kappa}^{\alpha / 2}\right\|_{0, \kappa}^{2}, \\
& \eta_{\alpha ; B_{\kappa}}^{* 2}:=\frac{h_{\kappa}^{2}}{N_{\kappa}^{2}}\left\|\left(-L_{\kappa}^{*} u_{N, h}^{*}+\lambda_{N, h}^{*} u_{N, h}^{*}\right) \Phi_{\kappa}^{\alpha / 2}\right\|_{0, \kappa}^{2} .
\end{aligned}
$$

Their second terms $\eta_{\alpha ; E_{\kappa}}^{2}, \eta_{\alpha ; E_{\kappa}}^{* 2}$ are the weighted element boundary residuals given by

$$
\begin{aligned}
& \eta_{\alpha ; E_{\kappa}}^{2}:=\sum_{e \subset \partial \kappa \cap \Omega} \frac{h_{e}}{2 N_{e}}\left\|D_{\kappa}\left[\frac{\partial u_{N, h}}{\partial n}\right] \Phi_{e}^{\alpha / 2}\right\|_{0, e}^{2}, \\
& \eta_{\alpha ; E_{\kappa}}^{* 2}:=\sum_{e \subset \partial \kappa \cap \Omega} \frac{h_{e}}{2 N_{e}}\left\|D_{\kappa}\left[\frac{\partial u_{N, h}^{*}}{\partial n}\right] \Phi_{e}^{\alpha / 2}\right\|_{0, e}^{2},
\end{aligned}
$$

where we denote the jump of the normal derivatives of $u_{N, h}$ and $u_{N, h}^{*}$ across the edges by $\left[\partial u_{N, h} / \partial n\right]$ and $\left[\partial u_{N, h}^{*} / \partial n\right]$, respectively. $h_{e}$ is the length of edge $e$. The weight functions $\Phi_{\kappa}$ and $\Phi_{e}$ are scaled transformations of the weight functions $\Phi_{\widehat{\kappa}}$ and $\Phi_{\widehat{e}}$; that is, if $F_{\kappa}$ is the element map for element $\kappa$ and $e$ is the image of the edge $\hat{e}$ under $F_{\kappa}$, then

$$
\Phi_{\kappa}=C_{\kappa} \Phi_{\widehat{\kappa}} \circ F_{\kappa}^{-1}, \quad \Phi_{e}=C_{e} \Phi_{\widehat{e}} \circ F_{\kappa}^{-1},
$$

where we choose $C_{\kappa}, C_{e}>0$, such that

$$
\int_{\kappa} \Phi_{\kappa} d x d y=\int_{\kappa} d x d y, \quad \int_{e} \Phi_{e} d s=\int_{e} d s .
$$

We define the global error indicators as follows:

$$
\begin{aligned}
\eta_{\alpha}^{2} & :=\sum_{\kappa \in K_{h}} \eta_{\alpha ; \kappa}^{2}, \\
\eta_{\alpha}^{* 2} & :=\sum_{\kappa \in K_{h}} \eta_{\alpha ; \kappa}^{* 2} .
\end{aligned}
$$


Theorem 12. Let $\alpha \in[0,1]$. Then there exists a constant $C>0$ independent of $h, \mathbf{N}$, and $\kappa$, such that

$$
\left.\begin{array}{rl}
\left\|u-u_{N, h}\right\|_{1, \Omega}^{2} \leq & C \sum_{\kappa \in K_{h}} N_{\kappa}^{2 \alpha} \eta_{\alpha ; \kappa}^{2} \\
+C \sum_{\kappa \in K_{h}}\left\{\frac{h_{\kappa}^{2}}{N_{\kappa}^{2}}\left\|L_{\kappa} u_{N, h}-L u_{N, h}\right\|_{0, \kappa}^{2}\right. \\
+\sum_{e \subset \partial \kappa \cap \Omega} \frac{h_{e}}{N_{e}}\left\|D-D_{\kappa}\right\|_{0, e}^{2} \\
\times\left\|\frac{\partial u_{N, h}}{\partial n}\right\|_{0, \infty, e}^{2}
\end{array}\right\}
$$

Proof. We denote $w:=u-u_{N, h}-I\left(u-u_{N, h}\right)$, where $I$ is $h p$ clément operator given by Lemma 8 . We derive from $H_{0}^{1}(\Omega)$ elliptic of $a(\cdot, \cdot)$ that

$$
\begin{aligned}
& C\left\|u-u_{N, h}\right\|_{1, \Omega}^{2} \leq a\left(u-u_{N, h}, w\right) \\
& +a\left(u-u_{N, h}, I\left(u-u_{N, h}\right)\right) \\
& =\lambda \int_{\Omega} u \bar{w}-a\left(u_{N, h}, w\right) \\
& +\int_{\Omega}\left(\lambda u-\lambda_{N, h} u_{N, h}\right) \\
& \times \overline{I\left(u-u_{N, h}\right)} \\
& =\int_{\Omega}\left(\lambda_{N, h} u_{N, h}\right) \bar{w} \\
& +\int_{\Omega}\left(\lambda u-\lambda_{N, h} u_{N, h}\right) \overline{u-u_{N, h}} \\
& -a\left(u_{N, h}, w\right), \\
& a\left(u_{N, h}, w\right)=\sum_{\kappa \in \kappa_{h}} \int_{\mathcal{K}} L u_{N, h} \bar{w} \\
& -\sum_{\kappa \in \kappa_{h}} \int_{\partial \kappa} D \frac{\partial u_{N, h}}{\partial n} \bar{w} \\
& =\sum_{\kappa \in \kappa_{h}} \int_{\kappa} L u_{N, h} \bar{w} \\
& -\frac{1}{2} \sum_{\kappa \in \kappa_{h}} \sum_{e \subset \partial \kappa \cap \Omega} \int_{e} D\left[\frac{\partial u_{N, h}}{\partial n}\right] \bar{w} .
\end{aligned}
$$

Therefore,

$$
\begin{aligned}
C \| u & -u_{N, h} \|_{1, \Omega}^{2} \\
\leq & \sum_{\kappa \in K_{h}} \int_{\mathcal{K}}\left(-L u_{N, h}+\lambda_{N, h} u_{N, h}\right) \bar{w}
\end{aligned}
$$

$$
\begin{gathered}
+\frac{1}{2} \sum_{\kappa \in K_{h}} \sum_{e \subset \partial \kappa \cap \Omega} \int_{e} D\left[\frac{\partial u_{N, h}}{\partial n}\right] \bar{w} \\
+\int_{\Omega}\left(\lambda u-\lambda_{N, h} u_{N, h}\right) \overline{\left(u-u_{N, h}\right)},
\end{gathered}
$$

which together with

$$
\begin{aligned}
\int_{e} D\left[\frac{\partial u_{N, h}}{\partial n}\right] \bar{w}= & \int_{e}\left(D-D_{\kappa}\right)\left[\frac{\partial u_{N, h}}{\partial n}\right] \bar{w} \\
& +\int_{e} D_{\kappa}\left[\frac{\partial u_{N, h}}{\partial n}\right] \bar{w} \\
\int_{\kappa}\left(-L u_{N, h}+\lambda_{N, h} u_{N, h}\right) \bar{w}= & \int_{\kappa}\left(-L_{\kappa} u_{N, h}+\lambda_{N, h} u_{N, h}\right) \bar{w} \\
& +\int_{\kappa}\left(L_{\kappa} u_{N, h}-L u_{N, h}\right) \bar{w}
\end{aligned}
$$

and using Cauchy-Schwartz inequality, the $h p$-clément interpolation estimates in Lemma 8 then yield

$$
\begin{aligned}
& \| u- u_{N, h} \|_{1, \Omega}^{2} \\
& \leq C\left\{\sum _ { \kappa \in K _ { h } } \left[\eta_{0 ; B_{\kappa}}^{2}+\eta_{0 ; E_{\kappa}}^{2}+\frac{h_{\kappa}^{2}}{N_{\kappa}^{2}}\left\|L_{\kappa} u_{N, h}-L u_{N, h}\right\|_{0, \kappa}^{2}\right.\right. \\
&\left.\left.\quad+\sum_{e \subset \partial \kappa \cap \Omega} \frac{h_{e}}{N_{e}}\left\|\left(D-D_{\kappa}\right)\left[\frac{\partial u_{N, h}}{\partial n}\right]\right\|_{0, e}^{2}\right]\right\}^{1 / 2} \\
& \times\left\|u-u_{N, h}\right\|_{1, \Omega}+C\left\|\lambda u-\lambda_{N, h} u_{N, h}\right\|_{0, \Omega}\left\|u-u_{N, h}\right\|_{0, \Omega} \cdot
\end{aligned}
$$

Using scaled transformation and setting $\alpha=0, \beta=\alpha$ in (61) and (62), we get $\eta_{0 ; E_{\kappa}} \leq C N_{\kappa}^{\alpha} \eta_{\alpha ; E_{\kappa}}$ and $\eta_{0 ; B_{\kappa}} \leq C N_{\kappa}^{\alpha} \eta_{\alpha ; B_{\kappa}}$; then this proof concludes.

For the adjoint eigenvalue problem, we still have the following.

Theorem 13. Let $\alpha \in[0,1]$. Then there exists a constant $C>0$ independent of $h, \mathbf{N}$, and $\kappa$, such that

$$
\left.\begin{array}{l}
\left\|u^{*}-u_{N, h}^{*}\right\|_{1, \Omega}^{2} \\
\leq C \sum_{\kappa \in K_{h}} N_{\kappa}^{2 \alpha} \eta_{\alpha ; \kappa}^{* 2} \\
+C \sum_{\kappa \in K_{h}}\left\{\frac{h_{\kappa}^{2}}{N_{\kappa}^{2}}\left\|L_{\kappa}^{*} u_{N, h}^{*}-L^{*} u_{N, h}^{*}\right\|^{2}\right. \\
+\sum_{e \subset \partial \kappa \cap \Omega} \frac{h_{e}}{N_{e}}\left\|D-D_{\kappa}\right\|_{0, e}^{2}\left\|\frac{\partial u_{N, h}^{*}}{\partial n}\right\|_{0, \infty, e}^{2}
\end{array}\right\}
$$


Lemma 14. Let $\alpha \in[0,1], \varepsilon>0$. Then there exists a constant $C(\varepsilon)>0$ independent of $h, \mathbf{N}$, and $\kappa$, such that

$$
\begin{aligned}
\eta_{\alpha ; B_{\kappa}}^{2} \leq C(\varepsilon)\{ & N_{\kappa}^{2(1-\alpha)}\left\|u-u_{N, h}\right\|_{1, \kappa}^{2} \\
& +N_{\kappa}^{\max \{1+2 \varepsilon-2 \alpha, 0\}} \frac{h_{\kappa}^{2}}{N_{\kappa}^{2}} \\
& \left.\times\left(\left\|\lambda_{N, h} u_{N, h}-\lambda u+L u_{N, h}-L_{\kappa} u_{N, h}\right\|_{0, k}^{2}\right)\right\} .
\end{aligned}
$$

Proof. We denote $v_{\kappa}:=\left(-L_{\kappa} u_{N, h}+\lambda_{N, h} u_{N, h}\right) \Phi_{\kappa}^{\alpha} \in H_{0}^{1}(\kappa)$ with $\alpha \in(0,1]$ and extend $v_{\kappa}$ to $\Omega$ by $v_{\kappa}=0$ on $\Omega \backslash \kappa$; then

$$
\begin{aligned}
\left\|v_{\kappa} \Phi_{\kappa}^{-\alpha / 2}\right\|_{0, \kappa}^{2}= & \int_{\mathcal{K}}\left(-L_{\kappa} u_{N, h}+\lambda_{N, h} u_{N, h}\right) \overline{v_{\kappa}} \\
= & -\int_{\mathcal{K}}\left(L_{\kappa} u_{N, h}\right) \overline{v_{\kappa}}+a\left(u, v_{\kappa}\right) \\
& +\int_{\mathcal{\kappa}}\left(\lambda_{N, h} u_{N, h}-\lambda u\right) \overline{v_{\kappa}} \\
= & a\left(u-u_{N, h}, v_{\kappa}\right) \\
& +\int_{\kappa}\left(\lambda_{N, h} u_{N, h}-\lambda u+L u_{N, h}-L_{\kappa} u_{N, h}\right) \overline{v_{\kappa}} \\
\leq & C\left\|u-u_{N, h}\right\|_{1, \kappa}\left|v_{\kappa}\right|_{1, \kappa} \\
& +\|\left(\lambda_{N, h} u_{N, h}-\lambda u\right. \\
& \left.+L u_{N, h}-L_{\kappa} u_{N, h}\right) \Phi_{\kappa}^{\alpha / 2} \|_{0, \kappa} \\
& \times\left\|v_{\kappa} \Phi_{\kappa}^{-\alpha / 2}\right\|_{0, \kappa} \cdot
\end{aligned}
$$

We consider the $H^{1}$ semi norm for $v_{\kappa}$. Using the polynomial inverse estimates (62)-(63) in Lemma 10, by transformation between the reference element $\widehat{\kappa}$ and $\kappa$, we find for $\alpha>1 / 2$ that

$$
\begin{aligned}
\left|v_{\kappa}\right|_{1, \kappa}^{2} \leq & 2 \int_{\mathcal{\kappa}} \Phi_{\kappa}^{2 \alpha}\left|\nabla\left(\lambda_{N, h} u_{N, h}-L_{\kappa} u_{N, h}\right)\right|^{2} \\
& +2 \int_{\mathcal{K}}\left|\nabla \Phi_{\kappa}^{\alpha}\right|^{2}\left|\lambda_{N, h} u_{N, h}-L_{\kappa} u_{N, h}\right|^{2} \\
\leq & C \frac{N_{\kappa}^{2(2-\alpha)}}{h_{\kappa}^{2}} \int_{\kappa} \Phi_{\kappa}^{\alpha}\left|\lambda_{N, h} u_{N, h}-L_{\kappa} u_{N, h}\right|^{2} \\
& +C \frac{1}{h_{\kappa}^{2}} \int_{\kappa} \Phi_{\kappa}^{2(\alpha-1)}\left|\lambda_{N, h} u_{N, h}-L_{\kappa} u_{N, h}\right|^{2} \\
\leq & C \frac{N_{\kappa}^{2(2-\alpha)}}{h_{\kappa}^{2}} \int_{\kappa} \Phi_{\kappa}^{\alpha}\left|\lambda_{N, h} u_{N, h}-L_{\kappa} u_{N, h}\right|^{2} \\
= & C N_{\kappa}^{2(1-\alpha)} \frac{N_{\kappa}^{2}}{h_{\kappa}^{2}}\left\|v_{\kappa} \Phi_{\kappa}^{-\alpha / 2}\right\|_{0, \kappa}^{2} .
\end{aligned}
$$

Note that (62) is applicable since $\alpha>1 / 2$ implies $2(\alpha-1)>$ -1 ; thus, we set $\beta=\alpha, \alpha=2(\alpha-1)$ in (62); then the third inequality above holds.
Since $\eta_{\alpha ; B_{\kappa}}=h_{\kappa} / N_{\kappa}\left\|v_{\kappa} \Phi_{\kappa}^{-\alpha / 2}\right\|_{0, \kappa}$, we obtain

$$
\begin{aligned}
& \eta_{\alpha ; B_{\kappa}} \leq C( N_{\kappa}^{1-\alpha}\left\|u-u_{N, h}\right\|_{1, \kappa} \\
&+\frac{h_{\kappa}}{N_{\kappa}} \| L u_{N, h}-L_{\kappa} u_{N, h} \\
&\left.\quad+\lambda_{N, h} u_{N, h}-\lambda u \|_{0, \kappa}\right) .
\end{aligned}
$$

To obtain an upper bound in the case of $0 \leq \alpha \leq 1 / 2$, we use the polynomial inverse estimate (62) in Lemma 10; for $\beta>$ $1 / 2$, we derive from (62) that

$$
\begin{aligned}
\frac{N_{\kappa}}{h_{\kappa}} \eta_{\alpha ; B_{\kappa}} & =\Phi_{\kappa}^{\alpha / 2}\left\|\left(\lambda_{N, h} u_{N, h}-L_{\kappa} u_{N, h}\right)\right\|_{0, \kappa} \\
& \leq C N_{\kappa}^{\beta-\alpha}\left\|\left(\lambda_{N, h} u_{N, h}-L_{\kappa} u_{N, h}\right) \Phi_{\kappa}^{\beta / 2}\right\|_{0, \kappa} \\
& =C N_{\kappa}^{\beta-\alpha} \frac{N_{\kappa}}{h_{\kappa}} \eta_{\beta ; B_{\kappa}} \\
\leq & C N_{\kappa}^{\beta-\alpha}\left(N_{\kappa}^{1-\beta} \frac{N_{\kappa}}{h_{\kappa}}\left\|u-u_{N, h}\right\|_{1, \kappa}\right. \\
& \left.\quad+\left\|\lambda_{N, h} u_{N, h}-\lambda u+L u_{N, h}-L_{\kappa} u_{N, h}\right\|_{0, \kappa}\right) .
\end{aligned}
$$

Setting $\beta=1 / 2+\varepsilon, \varepsilon>0$,

$$
\begin{aligned}
\eta_{\alpha ; B_{\kappa}} \leq C(\varepsilon)\{ & N_{\kappa}^{1-\alpha}\left\|u-u_{N, h}\right\|_{1, \kappa} \\
& +N_{\kappa}^{1 / 2+\varepsilon-\alpha} \frac{h_{\kappa}}{N_{\kappa}} \\
& \left.\times\left\|\lambda_{N, h} u_{N, h}-\lambda u+L u_{N, h}-L_{\kappa} u_{N, h}\right\|_{0, \kappa}\right\} .
\end{aligned}
$$

We obtain the desired result immediately from (83) and (85).

In order to obtain a local upper bound for the error indicator $\eta_{\alpha ; \kappa}$, we consider the edge residual term $\eta_{\alpha ; E_{\kappa}}$. we introduce the set

$$
\omega_{\kappa}:=\cup\left\{\kappa^{\prime} \mid \kappa^{\prime} \text { and } \kappa \text { share at least one edge }\right\} \text {. }
$$

Lemma 15. Let $\alpha \in[0,1], \varepsilon>0$. Then there exists a constant $C(\varepsilon)>0$ independent of $h, \mathbf{N}$, and $\kappa$, such that

$$
\begin{aligned}
\eta_{\alpha ; E_{\kappa}}^{2} \leq & C(\varepsilon) N_{\kappa}^{\max (1-2 \alpha+2 \varepsilon, 0)} \\
\times & \left\{N_{\kappa}\left\|u-u_{N, h}\right\|_{1, \omega_{\kappa}}^{2}+N_{\kappa}^{2 \varepsilon} \frac{h_{\kappa}^{2}}{N_{\kappa}^{2}}\right. \\
& \left.\times\left\|\lambda_{N, h} u_{N, h}-\lambda u+L u_{N, h}-L_{\kappa} u_{N, h}\right\|_{0, \omega_{\kappa}}^{2}\right\} .
\end{aligned}
$$


Proof. We will use weight functions on edge and a suitable extension operator. For a given element $\kappa$ with edge $e$, we choose the element $\kappa_{1}$ so that $\partial \kappa_{1} \cap \partial \kappa=e$. Denote $\overline{\kappa_{e}}:=$ $\overline{\kappa_{1}} \cup \bar{\kappa}$; we construct a function $w_{e} \in H_{0}^{1}\left(\kappa_{e}\right)$ with $\left.w_{e}\right|_{e}=$ $D_{\kappa}\left[\partial u_{N, h} / \partial n\right] \Phi_{e}^{\alpha}$ as follows.

Let $v_{\widehat{e}}=C_{e} D_{\kappa}\left[\partial u_{N, h} / \partial n\right] \Phi_{\widehat{e}}^{\alpha}\left(C_{e}\right.$ is defined by (71)). Using Lemma 11 , we extend $v_{\widehat{e}}$ to $\widehat{\kappa}$, where the polynomial $\pi$ corresponds to $C_{e} D_{\kappa}\left[\partial u_{N, h} / \partial n\right]$. Define $\left.w_{e}\right|_{\kappa}$ and $\left.w_{e}\right|_{\kappa_{1}}$ as the affine transformation of $v_{\widehat{e}}$ in $\widehat{\kappa}$; Thus, $w_{e}$ is a piecewise $H^{1}$ function. From (64), we know $w_{e}$ vanishes on $\partial \kappa_{e}$; Therefore, $w_{e} \in H_{0}^{1}\left(\kappa_{e}\right)$. It is trivial to extend $w_{e}$ to $\Omega$, such that $w_{e}=0$ in $\Omega \backslash \kappa_{e}$. We find

$$
\begin{aligned}
\| D_{\kappa} & {\left[\frac{\partial u_{N, h}}{\partial n}\right] \Phi_{e}^{\alpha / 2} \|_{0, e}^{2} } \\
= & \int_{e} D\left[\frac{\partial u_{N, h}}{\partial n}\right] \overline{w_{e}}+\int_{e}\left(D_{\kappa}-D\right)\left[\frac{\partial u_{N, h}}{\partial n}\right] \overline{w_{e}} \\
= & \int_{\mathcal{K}_{e}} L u_{N, h} \overline{w_{e}}-a\left(u_{N, h}, w_{e}\right) \\
& +\int_{e}\left(D_{\kappa}-D\right)\left[\frac{\partial u_{N, h}}{\partial n}\right] \overline{w_{e}} \\
= & \int_{\mathcal{K}_{e}}\left(L u_{N, h}-\lambda u\right) \overline{w_{e}}+a\left(u-u_{N, h}, w_{e}\right) \\
& +\int_{e}\left(D_{\kappa}-D\right)\left[\frac{\partial u_{N, h}}{\partial n}\right] \overline{w_{e}} \\
\leq & \left\|L u_{N, h}-\lambda u\right\|_{0, \kappa_{e}}\left\|w_{e}\right\|_{0, \kappa_{e}} \\
& +C\left\|u-u_{N, h}\right\|_{1, \kappa_{e}}\left|w_{e}\right|_{1, \kappa_{e}} \\
& +\left\|\frac{\left(D_{\kappa}-D\right)}{D_{\kappa}}\right\|_{0, \infty, e}\left\|_{\kappa}\left[\frac{\partial u_{N, h}}{\partial n}\right] \Phi_{e}^{\alpha / 2}\right\|_{0, e}^{2}
\end{aligned}
$$

Therefore,

$$
\begin{aligned}
\left\|D_{\kappa}\left[\frac{\partial u_{N, h}}{\partial n}\right] \Phi_{e}^{\alpha / 2}\right\|_{0, e}^{2} \\
\leq C\left\|L u_{N, h}-\lambda u\right\|_{0, \kappa_{e}}\left\|w_{e}\right\|_{0, \kappa_{e}} \\
\quad+C\left\|u-u_{N, h}\right\|_{1, \kappa_{e}}\left|w_{e}\right|_{1, \kappa_{e}} .
\end{aligned}
$$

We consider the case of $\alpha \in(1 / 2,1]$ first. Using the affine equivalence and (65)-(66) in Lemma 11, we obtain the upper bounds for $\left\|w_{e}\right\|_{0, \kappa_{e}}$ and $\left|w_{e}\right|_{1, \kappa_{e}}$ as follows:

$$
\begin{aligned}
\left|w_{e}\right|_{1, \kappa_{e}}^{2} \leq & C \frac{1}{h_{\kappa}}\left(\varepsilon N_{\kappa}^{2(2-\alpha)}+\varepsilon^{-1}\right) \\
\times & \left\|D_{\kappa}\left[\frac{\partial u_{N, h}}{\partial n}\right] \Phi_{e}^{\alpha / 2}\right\|_{0, e}^{2}, \\
\left\|w_{e}\right\|_{0, \kappa_{e}} \leq & C h_{\kappa} \varepsilon\left\|D_{\kappa}\left[\frac{\partial u_{N, h}}{\partial n}\right] \Phi_{e}^{\alpha / 2}\right\|_{0, e}^{2} .
\end{aligned}
$$

It follows from (89)-(90) that

$$
\begin{aligned}
&\left\|D_{\kappa}\left[\frac{\partial u_{N, h}}{\partial n}\right] \Phi_{e}^{\alpha / 2}\right\|_{0, e} \\
& \leq C\left\{\left(\frac{1}{h_{\kappa}}\left(\varepsilon N_{\kappa}^{2(2-\alpha)}+\varepsilon^{-1}\right)\right)^{1 / 2}\right. \\
& \times\left.\times u-u_{N, h}\left\|_{1, \kappa_{e}}+\left(h_{\kappa} \varepsilon\right)^{1 / 2}\right\|\left\|L u_{N, h}-\lambda u\right\|_{0, \kappa_{e}}\right\} .
\end{aligned}
$$

By the definition of $\eta_{\alpha ; E_{\kappa}}^{2}$ and setting $\alpha=0$ in Lemma 14, we get

$$
\begin{aligned}
\| L_{\kappa} u_{N, h}- & \lambda_{N, h} u_{N, h} \|_{0, k}^{2} \\
\leq C(\varepsilon) & \left\{\frac{N_{\kappa}^{4}}{h_{\kappa}^{2}}\left\|u-u_{N, h}\right\|_{1, k}^{2}+N_{\kappa}^{1+2 \varepsilon}\right. \\
& \left.\times\left\|\lambda_{N, h} u_{N, h}-\lambda u+L u_{N, h}-L_{\kappa} u_{N, h}\right\|_{0, k}^{2}\right\},
\end{aligned}
$$

by the triangle inequality

$$
\begin{aligned}
& \left\|L u_{N, h}-\lambda u\right\|_{0, \kappa_{e}} \\
& \leq\left\|L_{\kappa} u_{N, h}-\lambda_{N, h} u_{N, h}\right\|_{0, \kappa_{e}} \\
& \quad+\left\|\lambda_{N, h} u_{N, h}-\lambda u+L u_{N, h}-L_{\kappa} u_{N, h}\right\|_{0, \kappa_{e}} .
\end{aligned}
$$

Combining the three inequalities above and summing, we have

$$
\begin{aligned}
\eta_{\alpha ; E_{\kappa}}^{2} \leq & C\left\{\frac{1}{N_{\kappa}}\left(\varepsilon N_{\kappa}^{2(2-\alpha)}+\varepsilon^{-1}\right)+N_{\kappa}^{3} \varepsilon\right\} \\
& \times\left\|u-u_{N, h}\right\|_{1, \omega_{\kappa}}^{2}+C \varepsilon N_{\kappa}^{2(1+\varepsilon)} \frac{h_{\kappa}^{2}}{N_{\kappa}^{2}} \\
& \times\left\|\lambda_{N, h} u_{N, h}-\lambda u+L u_{N, h}-L_{\kappa} u_{N, h}\right\|_{0, \omega_{\kappa}}^{2} .
\end{aligned}
$$

Setting $\varepsilon=1 / N_{\kappa}^{2}$ in the above inequality yields the assertion for $\alpha>1 / 2$. For the case of $\alpha \in[0,1 / 2]$, we set $\beta=1 / 2+\varepsilon$, use (62) in Lemma 10 to get $\eta_{\alpha ; E_{\kappa}} \leq N_{\kappa}^{\beta-\alpha} \eta_{\beta ; E_{\kappa}}$, and find the desired result.

Combining Lemmas 14 and 15, we obtain the following theorem.

Theorem 16. Let $\alpha \in[0,1], \varepsilon>0$. Then there exists a constant $C>0$ independent of $h, \mathbf{N}$, and $\kappa$, such that

$$
\begin{aligned}
\eta_{\alpha ; \kappa}^{2} \leq & C(\varepsilon) N_{\kappa}^{\max (1-2 \alpha+2 \varepsilon, 0)} \\
\times & \left\{N_{\kappa}\left\|u-u_{N, h}\right\|_{1, \omega_{\kappa}}^{2}+N_{\kappa}^{2 \varepsilon} \frac{h_{\kappa}^{2}}{N_{\kappa}^{2}}\right. \\
& \left.\quad \times\left\|\lambda_{N, h} u_{N, h}-\lambda u+L u_{N, h}-L_{\kappa} u_{N, h}\right\|_{0, \omega_{\kappa}}^{2}\right\} .
\end{aligned}
$$

Similarly, we have Theorem 17. 
Theorem 17. Let $\alpha \in[0,1], \varepsilon>0$. Then there exists a constant $C>0$ independent of $h, \mathbf{N}$, and $\kappa$, such that

$$
\begin{aligned}
\eta_{\alpha ; \kappa}^{* 2} \leq & C(\varepsilon) N_{\kappa}^{\max (1-2 \alpha+2 \varepsilon, 0)} \\
\times & \left\{N_{\kappa}\left\|u^{*}-u_{N, h}^{*}\right\|_{1, \omega_{\kappa}}^{2}+N_{\kappa}^{2 \varepsilon} \frac{h_{\kappa}^{2}}{N_{\kappa}^{2}}\right. \\
& \left.\times\left\|\lambda_{N, h}^{*} u_{N, h}^{*}-\lambda^{*} u^{*}+L^{*} u_{N, h}^{*}-L_{\kappa}^{*} u_{N, h}^{*}\right\|_{0, \omega_{\kappa}}^{2}\right\} .
\end{aligned}
$$

In order to estimate bounds of $\left|\lambda-\lambda_{N, h}\right|$, we also need Lemma 18 (see $[8,10]$ ).

Lemma 18. Let $(\lambda, u)$ be an eigenpair of $(24)$, and let $\left(\lambda^{*}=\right.$ $\left.\bar{\lambda}, u^{*}\right)$ be the associated eigenpair of the adjoint problem (31). Then for all $w, w^{*} \in H_{0}^{1}(\Omega),\left(w, w^{*}\right) \neq 0$,

$$
\begin{aligned}
& \frac{a\left(w, w^{*}\right)}{\left(w, w^{*}\right)}-\lambda \\
& =\frac{a\left(w-u, w^{*}-u^{*}\right)}{\left(w, w^{*}\right)}-\lambda \frac{\left(w-u, w^{*}-u^{*}\right)}{\left(w, w^{*}\right)} .
\end{aligned}
$$

Theorem 19. Under the assumptions of Theorem 7, we assume that $D, \mathbf{b}$, and $c$ are smooth enough, and let $\alpha \in[0,1]$. Then there exists an eigenpair $(\lambda, u)$ of $(24)$, such that

$$
\begin{gathered}
\left\|u-u_{N, h}\right\|_{1, \Omega} \leq C\left(\sum_{\kappa \in K_{h}} N_{\kappa}^{2 \alpha} \eta_{\alpha ; \kappa}^{2}\right)^{1 / 2}, \\
\eta_{\alpha ; \kappa}^{2} \leq C(\varepsilon) N_{\kappa}^{\max (2-2 \alpha+2 \varepsilon, 1)}\left\|u-u_{N, h}\right\|_{1, \omega_{\kappa}}^{2} .
\end{gathered}
$$

Further let the ascent of $\lambda_{N, h}$ be $l=1$, and let $\left(\lambda_{N, h}^{*}, u_{N, h}^{*}\right)$ be the corresponding adjoint eigenpair of (32), then there exists an adjoint eigenpair $\left(\lambda^{*}, u^{*}\right)$ of $(31)$, such that

$$
\left|\lambda_{N, h}-\lambda\right| \leq C\left(\sum_{\kappa \in K_{h}} N_{\kappa}^{2 \alpha} \eta_{\alpha ; \kappa}^{2}\right)^{1 / 2}\left(\sum_{\kappa \in K_{h}} N_{\kappa}^{2 \alpha} \eta_{\alpha ; \kappa}^{* 2}\right)^{1 / 2}
$$

Particularly, if the eigenvalue problem (23) is symmetric (i.e., $\mathbf{b}=0$ ), then

$$
C C(\varepsilon)^{-1} \sum_{\kappa \in K_{h}} N_{\kappa}^{\min (2 \alpha-2-2 \varepsilon,-1)} \eta_{\alpha ; \kappa}^{2} \leq\left|\lambda_{N, h}-\lambda\right|
$$

Proof. We know from the assumption $D, c \in H^{t_{1}}(\kappa), \mathbf{b} \in$ $\left(H^{t_{1}}(\kappa)\right)^{2}$. By the interpolation error estimates (14) and (15), we have

$$
\left\|L_{\kappa} u_{N, h}-L u_{N, h}\right\|_{0, \kappa} \leq C h_{\kappa}^{\min \left(N_{\kappa}+1, t_{1}\right)-1} N_{\kappa}^{-t_{1}+1} .
$$

From $D \in H^{t_{1}}(\kappa)$, we know that $D \in H^{t_{1}-1 / 2}(e)$. By the interpolation error estimate on edge of element (see formula (5.4.42) in [2]), we get

$$
\left\|D-D_{\kappa}\right\|_{0, e} \leq C h_{e}^{\min \left(N_{\kappa}+1, t_{1}-1 / 2\right)} N_{\kappa}^{-t_{1}+1 / 2} .
$$

Note that the formula (51) gives the optimal orders of convergence; thus, we deduce that the second and third terms on the right side of (74) are higher order infinitesimals. We derive from (52) and (53), and $N=N_{\kappa}$, that

$$
\begin{aligned}
\| \lambda u & -\lambda_{N, h} u_{N, h} \|_{0, \Omega} \\
\leq & \left|\lambda-\lambda_{N, h}\right|\|u\|_{0, \Omega} \\
& +\left|\lambda_{N, h}\right|\left\|u-u_{N, h}\right\|_{0, \Omega} \\
\leq & \frac{C h^{\tau_{1}+\tau_{2}-2}}{N^{t_{1}+t_{2}-2}}+\frac{C h^{r_{2}+\tau_{1}-1}}{N^{r_{2}+t_{1}-1}} \leq \frac{C h^{r_{2}+\tau_{1}-1}}{N^{r_{2}+t_{1}-1}} .
\end{aligned}
$$

Therefore, the fourth term on the right side of (74) is also a higher order infinitesimal. Up to higher order terms, we get (98). We ignore higher order infinitesimals in (95) and get (99). From Lemma 4 in [10], we know that $\left(u_{N, h}, u_{N, h}^{*}\right)=1$ and $u_{N, h}^{*}$ is uniformly bounded with $h$ and $N$. By the same argument of (98), we can deduce that

$$
\left\|u^{*}-u_{N, h}^{*}\right\|_{1, \Omega}^{2} \leq C \sum_{\kappa \in K_{h}} N_{\kappa}^{2 \alpha} \eta_{\alpha ; \kappa}^{* 2} .
$$

From (97), we have

$$
\begin{aligned}
& \frac{a\left(u_{N, h}, u_{N, h}^{*}\right)}{\left(u_{N, h}, u_{N, h}^{*}\right)}-\lambda \\
& =\frac{a\left(u_{N, h}-u, u_{N, h}^{*}-u^{*}\right)}{\left(u_{N, h}, u_{N, h}^{*}\right)} \\
& -\lambda \frac{\left(u_{N, h}-u, u_{N, h}^{*}-u^{*}\right)}{\left(u_{N, h}, u_{N, h}^{*}\right)} ;
\end{aligned}
$$

that is,

$$
\begin{aligned}
\lambda_{N, h}-\lambda= & a\left(u_{N, h}-u, u_{N, h}^{*}-u^{*}\right) \\
& -\lambda\left(u_{N, h}-u, u_{N, h}^{*}-u^{*}\right) .
\end{aligned}
$$

Substituting (98) and (105) into the above equality, we obtain (100). then

If the eigenvalue problem (23) is symmetric (i.e., $\mathbf{b}=0$ ),

$$
\begin{aligned}
\lambda_{N, h}-\lambda= & a\left(u_{N, h}-u, u_{N, h}-u\right) \\
& -\lambda\left(u_{N, h}-u, u_{N, h}-u\right) .
\end{aligned}
$$

Up to higher order term $\lambda\left(u_{N, h}-u, u_{N, h}-u\right)$, by (99) we get (101).

Remark 20. Babuška and Osborn [17] have discussed hp finite element approximation with simplex partition for eigenvalue problems. Obviously, the Interpolation estimates (14) and (15) hold for hp finite element with simplex partition (see [24]). Therefore, our theoretical results of spectral methods and spectral methods for eigenvalue problems, which have been discussed in Sections 3 and 4, hold for hp finite element with simplex partition. 
TABLE 1: Errors of LGL-SM, modal, and Eq-SM for 1st eigenvalue.

\begin{tabular}{lcccc}
\hline$N$ & DOF & $\begin{array}{c}\text { LGL-SM } \\
\lambda_{1}\end{array}$ & $\begin{array}{c}\text { Modal-SM } \\
\lambda_{1}\end{array}$ & $\begin{array}{c}\text { Eq-SM } \\
\lambda_{1}\end{array}$ \\
\hline 4 & 9 & $5.19 E+00$ & $5.19 E+00$ & $5.19 E+00$ \\
5 & 16 & $4.51 E-01$ & $4.51 E-01$ & $4.51 E-01$ \\
6 & 25 & $7.68 E-03$ & $7.68 E-03$ & $7.68 E-03$ \\
7 & 36 & $1.07 E-05$ & $1.07 E-05$ & $1.07 E-05$ \\
8 & 49 & $1.21 E-05$ & $1.21 E-05$ & $1.21 E-05$ \\
9 & 64 & $9.16 E-07$ & $9.16 E-07$ & $9.16 E-07$ \\
10 & 81 & $2.46 E-08$ & $2.46 E-08$ & $2.48 E-08$ \\
11 & 100 & $2.91 E-10$ & $2.91 E-10$ & $4.35 E-09$ \\
12 & 121 & $9.31 E-13$ & $1.06 E-12$ & $2.79 E-08$ \\
13 & 144 & $5.68 E-14$ & $1.28 E-13$ & $1.41 E-07$ \\
14 & 169 & $2.84 E-14$ & $1.28 E-13$ & $2.28 E-06$ \\
15 & 196 & $7.82 E-14$ & $2.13 E-14$ & $3.60 E-05$ \\
\hline
\end{tabular}

\section{Numerical Experiments}

In this section, we simply denote spectral methods, spectral element methods, and finite element methods with SM, SEM, and FEM, respectively. And spectral methods with equidistant nodal basis, modal basis, and LGL nodal basis are replaced by Eq-SM, Modal-SM, and LGL-SM, respectively. Note that all these methods employ the tensorial basis.

In our experiment, we compute $1 /\left|\left(u_{N, h}, u_{N, h}^{*}\right)\right|$ as condition number for simple eigenvalue (see Remark 2.1 in [25]), where $u_{N, h}$ and $u_{N, h}^{*}$ are eigenfunctions of eigenvalue problem (25) and its adjoint problem (32) normalized with $\|\cdot\|_{0, \Omega}$, respectively.

5.1. Example 1. Consider the nonsymmetric eigenvalue problem

$$
\begin{gathered}
-\Delta u+10 u_{x}+u_{y}=\lambda u, \quad \text { in } \Omega=(0,1)^{2}, \\
u=0, \quad \text { on } \partial \Omega .
\end{gathered}
$$

The first eigenvalue of (109) $\lambda_{1}=101 / 4+2 \pi^{2}$ is a simple eigenvalue. And the corresponding eigenfunctions are sufficiently smooth.

5.1.1. Comparisons between LGL-SM, Modal, and Eq-SM. Figure 1 shows that the condition numbers of the first eigenvalue for LGL-SM, Modal-SM, and Eq-SM coincide with each other at the beginning but perform abnormally with $N>19$ for Eq-SM. Table 1 tells us that when $N>11$, the accuracy of first eigenvalue obtained by Eq-SM is not as good as obtained that by LGL-SM and Modal-SM. When $N=15$, the error of the first eigenvalues obtained by Eq-SM is greater than $1 \mathrm{E}-5$; however, the order of the magnitude of errors for LGL-SM and Modal-SM still keeps below 1E-13. The best result of first eigenvalue error for Eq-SM is merely $1 \mathrm{E}-9$ or so.

5.1.2. LGL-SM and Modal-SM versus hp-SEM. Tables 1 and 2 indicate that increasing the polynomial degree $N$ or

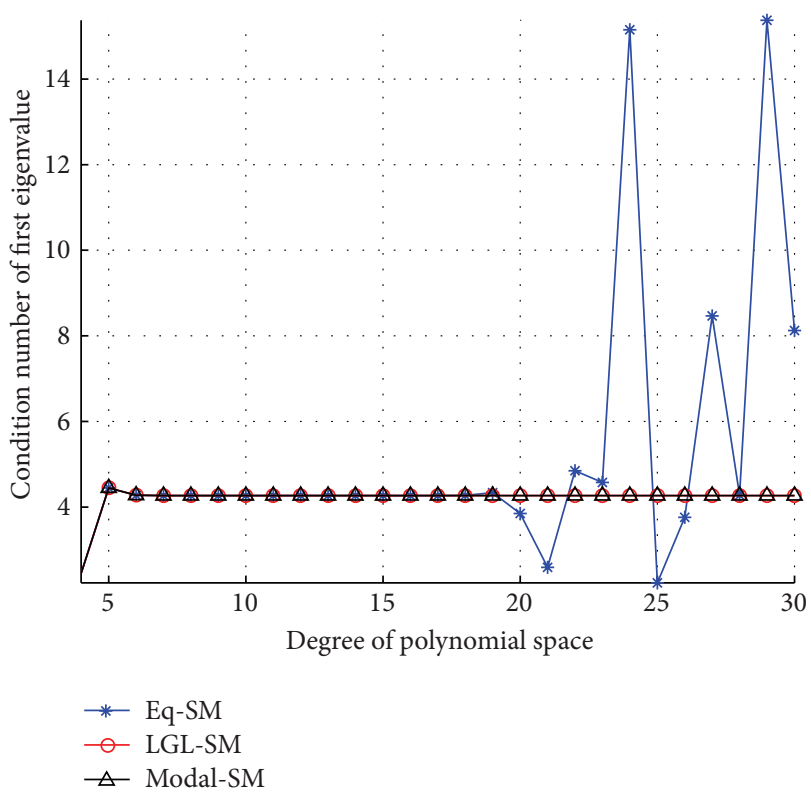

Figure 1: Condition number of first eigenvalue for SM.

decreasing the mesh fineness $h$ can decrease the errors of the first eigenvalue. But it is expensive to increase polynomial degree and decrease mesh fineness $h$ at the same time. For $h=1 / 4$ and $h=1 / 16$, we obtain from Table 2 the first eigenvalue errors $2.8 E-14$ and $1.3 E-13$ and the corresponding degree of freedom 1225 and 6241 for hp-SEM, respectively, Whereas from Table 1 , to reach this accuracy, LGL-SM and Modal-SM should merely perform the interpolation approximations with polynomial degree bi-14 and bi-13 or so, and the corresponding degrees of freedom are merely 169 and 144, respectively. Therefore, we conclude that LGL-SM and Modal-SM are highly accurate and efficient for this kind of nonsymmetric eigenvalue problems.

In Figure 2 from [9], when the degree of freedom is up to 1000 , the error of linear FEM is about 1E-2; the function value recovery techniques in [9] obviously improves the accuracy up to $1 E-5$. Comparing Tables 1 and 2 in this paper with Figure 2 in [9], we can also find the advantages of LGL-SM, Modal$\mathrm{SM}$, and hp-SEM over the function value recovery techniques for FEM given by [9] from accuracy and degree of freedom.

5.1.3. $h p$-SEM versus $h p$-FEM. From Table 4, we find that the condition number of the first eigenvalue for hp-version methods (hp-SEM and hp-FEM) stays at 4.27. It is indicated from Tables 2 and 3 that, when $N$ is greater than 7, compared with hp-SEM, the errors of hp-FEM tend to become large, whereas the errors of hp-SEM still keep stable or even stay a decreasing tendency; however, this phenomenon is not apparent for $h=1 / 2$.

Remark 21. Condition numbers of 1st eigenvalue for hp-FEM (not listed in Table 4) are almost the same to those for hpSEM. 
TABLE 2: Errors and DOF of hp-SEM for the first eigenvalue.

\begin{tabular}{|c|c|c|c|c|c|c|c|c|}
\hline \multirow{2}{*}{$N$} & \multicolumn{2}{|c|}{$h=1 / 2$} & \multicolumn{2}{|c|}{$h=1 / 4$} & \multicolumn{2}{|c|}{$h=1 / 8$} & \multicolumn{2}{|c|}{$h=1 / 16$} \\
\hline & Error & DOF & Error & DOF & Error & DOF & Error & DOF \\
\hline 2 & $5.18 E+00$ & 9 & $2.54 E-01$ & 49 & $1.50 E-02$ & 225 & $9.00 E-04$ & 961 \\
\hline 3 & $7.00 E-03$ & 25 & $6.10 E-04$ & 121 & $1.20 E-05$ & 529 & $1.90 E-07$ & 2209 \\
\hline 4 & $8.40 E-03$ & 49 & $2.60 E-05$ & 225 & $9.70 E-08$ & 961 & $3.70 E-10$ & 3969 \\
\hline 5 & $1.64 E-04$ & 81 & $1.60 E-07$ & 361 & $1.50 E-10$ & 1521 & $1.30 E-13$ & 6241 \\
\hline 6 & $4.10 E-07$ & 121 & $2.30 E-11$ & 529 & $9.90 E-13$ & 2209 & $3.60 E-12$ & 9025 \\
\hline 7 & $3.10 E-08$ & 169 & $1.70 E-12$ & 729 & $3.10 E-13$ & 3025 & $1.60 E-12$ & 12321 \\
\hline 8 & $1.90 E-10$ & 225 & $1.90 E-13$ & 961 & $2.10 E-12$ & 3969 & $4.80 E-12$ & 16129 \\
\hline 9 & $5.50 E-13$ & 289 & $2.80 E-14$ & 1225 & $6.00 E-13$ & 5041 & $1.10 E-12$ & 20449 \\
\hline 10 & $3.80 E-13$ & 361 & $1.10 E-12$ & 1521 & $4.40 E-12$ & 6241 & $1.50 E-11$ & 25281 \\
\hline
\end{tabular}

TABLE 3: Errors of hp-FEM for the first eigenvalue.

\begin{tabular}{lcccc}
\hline$N$ & $h=1 / 2$ & $h=1 / 4$ & $h=1 / 8$ & $h=1 / 16$ \\
\hline 3 & $7.00 E-03$ & $6.10 E-04$ & $1.20 E-05$ & $1.90 E-07$ \\
4 & $8.40 E-03$ & $2.60 E-05$ & $9.70 E-08$ & $3.70 E-10$ \\
5 & $1.60 E-04$ & $1.60 E-07$ & $1.50 E-10$ & $1.30 E-12$ \\
6 & $4.10 E-07$ & $2.40 E-11$ & $3.60 E-13$ & $8.60 E-12$ \\
7 & $3.10 E-08$ & $6.10 E-12$ & $1.30 E-11$ & $3.00 E-11$ \\
8 & $1.80 E-10$ & $3.10 E-11$ & $2.30 E-10$ & $2.10 E-10$ \\
9 & $7.50 E-11$ & $3.40 E-11$ & $6.80 E-10$ & $7.40 E-10$ \\
10 & $2.50 E-11$ & $9.90 E-10$ & $8.70 E-09$ & $6.60 E-09$ \\
11 & $2.00 E-09$ & $9.60 E-09$ & $8.90 E-09$ & $5.40 E-07$ \\
\hline
\end{tabular}

TABLE 4: Condition number of first eigenvalue for hp-SEM.

\begin{tabular}{ccccc}
\hline$N$ & $h=1 / 2$ & $h=1 / 4$ & $h=1 / 8$ & $h=1 / 16$ \\
\hline 3 & 4.284381324 & 4.270132842 & 4.269625046 & 4.269615821 \\
4 & 4.267343095 & 4.269607452 & 4.269615638 & 4.26961567 \\
5 & 4.269636446 & 4.269615725 & 4.26961567 & 4.26961567 \\
6 & 4.269619135 & 4.26961567 & 4.26961567 & 4.26961567 \\
7 & 4.269615617 & 4.26961567 & 4.26961567 & 4.26961567 \\
8 & 4.26961567 & 4.26961567 & 4.26961567 & 4.26961567 \\
9 & 4.26961567 & 4.26961567 & 4.26961567 & 4.26961567 \\
\hline
\end{tabular}

\subsubsection{Validity of the Error Indicator. Denote}

$$
\psi_{\alpha}=\left(\sum_{\kappa \in K_{h}} N_{\kappa}^{2 \alpha} \eta_{\alpha ; \kappa}^{2}\right)^{1 / 2}\left(\sum_{\kappa \in K_{h}} N_{\kappa}^{2 \alpha} \eta_{\alpha ; \kappa}^{* 2}\right)^{1 / 2}
$$

From Theorem 19, we know that $\psi_{\alpha}$ is a reliable error indicator for $\lambda_{N, h}$. We choose $\psi_{0}$ (setting $\alpha=0$ in (110)) as a posteriorii error indicator.

In Figures 2 and 3, we denote the true error and est. error with $\left|\lambda_{N, h}-\lambda\right|$ and $\psi_{0}$, respectively.

As is depicted in Figure 2, when the polynomial degree $N \leq 12$, the error indicator $\psi_{0}$ can properly estimate the true errors of LGL-SM for the first eigenvalue, however, also slightly underestimate the true errors. It is easy to see that $\psi_{0}$ shows almost the same algebraic decay as the true error with the polynomial degree $N(\leq 12)$ increasing. Nevertheless, the error indicator $\psi_{0}$ cannot approximate the true errors if $N$
TABLE 5: The Approximate eigenvalues and indicator $\psi_{0}$ of P-SEM.

\begin{tabular}{lcc}
\hline$N$ & $\lambda_{N, h}$ & $\psi_{0}$ \\
\hline 3 & 28.56900 & $2.72 E+01$ \\
4 & 31.99175 & $3.49 E+00$ \\
5 & 34.82082 & $2.25 E-01$ \\
6 & 34.65087 & $1.31 E-02$ \\
7 & 34.65057 & $3.32 E-03$ \\
8 & 34.64765 & $1.92 E-03$ \\
9 & 34.64567 & $1.22 E-03$ \\
10 & 34.64432 & $8.11 E-04$ \\
11 & 34.64335 & $5.62 E-04$ \\
12 & 34.64265 & $4.02 E-04$ \\
13 & 34.64212 & $2.95 E-04$ \\
14 & 34.64171 & $2.22 E-04$ \\
15 & 34.64139 & $1.71 E-04$ \\
16 & 34.64114 & $1.33 E-04$ \\
17 & 34.64094 & $1.06 E-04$ \\
18 & 34.64078 & $8.49 E-05$ \\
\hline
\end{tabular}

is large enough, which is caused by round-off errors derived from the bad condition number of eigenvalue. In Figure 3, we give the comparison between the error indicator $\psi_{0}$ and the true errors for hp-SEM.

5.2. Example 2. Consider the nonsymmetric eigenvalue problem

$$
\begin{aligned}
-\Delta u+10 u_{x} & =\lambda u, \quad \text { in } \Omega=\frac{(-1,1)^{2}}{(0,1)^{2}}, \\
u & =0, \quad \text { on } \partial \Omega .
\end{aligned}
$$

A reference value for the first eigenvalue (simple eigenvalue) of (111) is 34.6397 given by [5]. And the corresponding eigenfunctions have the singularity at the origin. Next, we shall compare the relevant numerical results between P-SEM and the other methods adopted in this paper. Note that here and hereafter P-version methods are for the fixed mesh fineness $h=1$. Table 5 lists part data of the approximate eigenvalues computed by P-SEM and the corresponding error indicator $\psi_{0}$ for reference. 


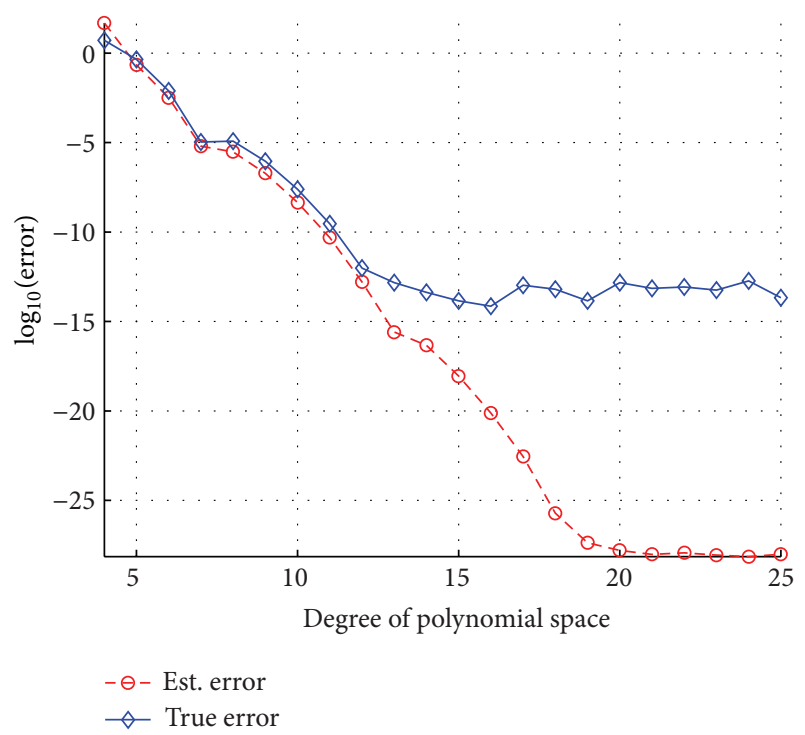

FIGURE 2: The Error indicator $\psi_{0}$ of LGL-SM.

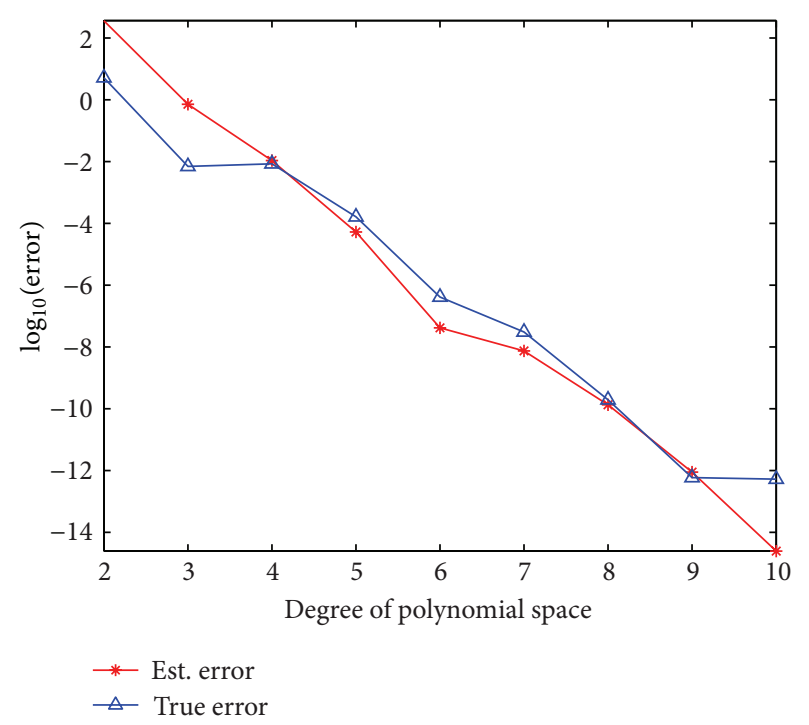

Figure 3: The Error indicator $\psi_{0}$ of hp-SEM $(h=1 / 2)$.

5.2.1. Stability of P-Version Methods. Figure 4 indicates that the eigenvalues computed by P-FEM will not seriously deviate from the results computed by P-SEM until the interpolation polynomial degree $N$ is up to 19 . This phenomenon coincides with the abnormity of condition number of first eigenvalue for P-FEM (see Figure 5). The reason is that the singularities of the eigenfunctions limit the accuracy of both kinds of methods; this is slightly different from the case of the eigenvalue problem with the sufficiently smooth eigenfunctions.

5.2.2. P-SEM versus Other Methods. By calculations, we find that, in the case of the linear FEM, for fixed mesh fineness $h=1 / 256$, the approximate eigenvalue is 34.6403 with degree of freedom up to 195585 . But P-SEM with the polynomial degree bi-22 can reach this accuracy, and the corresponding

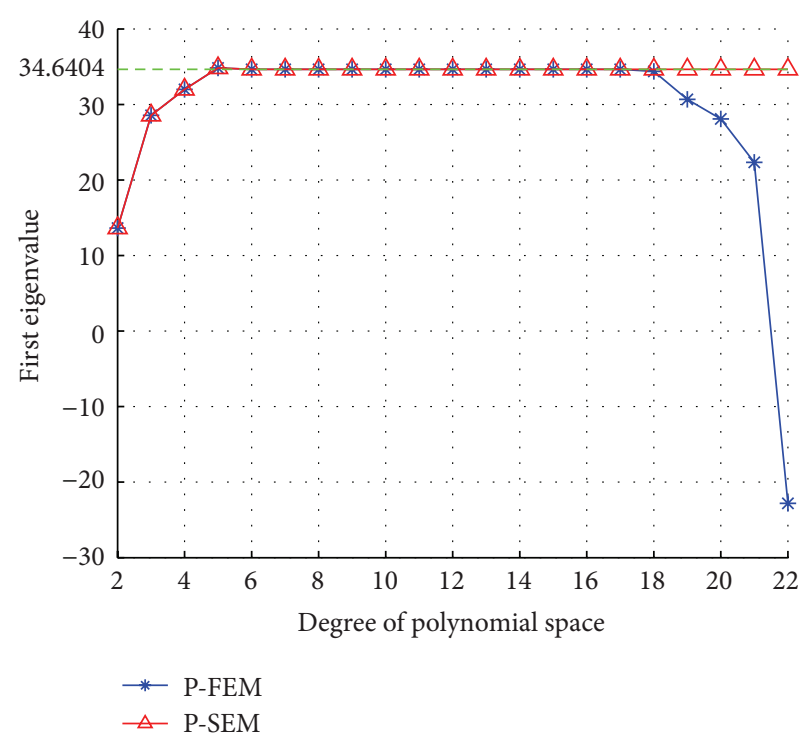

Figure 4: The Approximate 1st eigenvalue of P-SEM and P-FEM.

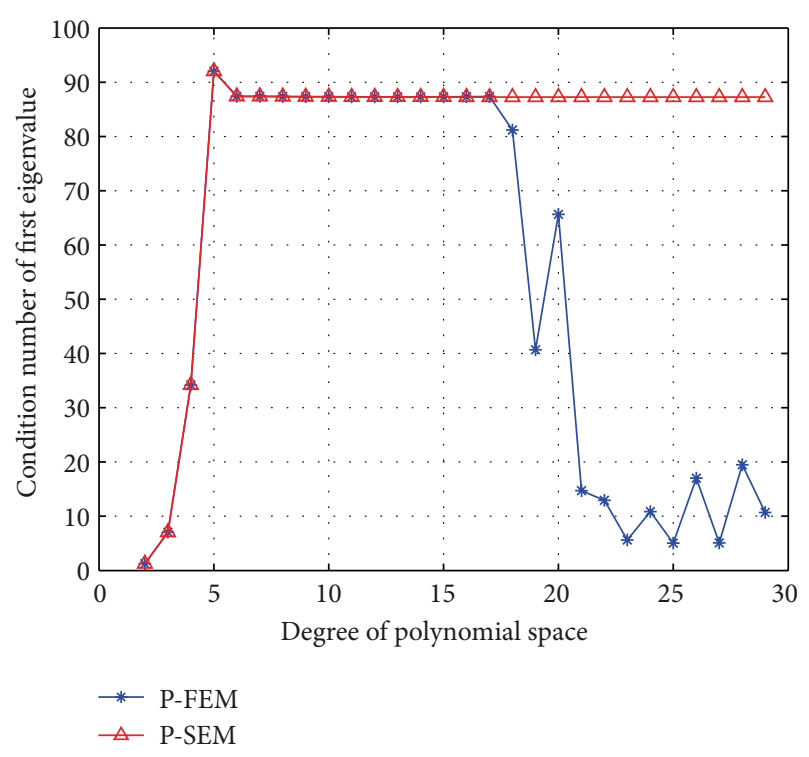

FIGURE 5: Condition number of first eigenvalue for P-SEM and PFEM.

degree of freedom is merely 1365 . Compared with the linear FEM, hp-SEM can obtain a higher accuracy with less degrees of freedom as follows: for fixed $h=1 / 16$ and $N=10$, the approximate eigenvalue is 34.63984 with degree of freedom 76161 but P-SEM with polynomial degree bi-44 can reach this accuracy. Therefore, P-SEM is more efficient for the eigenvalue problems with the singular solutions than the other methods.

\section{Acknowledgments}

This work was supported by the National Natural Science Foundation of China (Grant no. 11161012) and the Educational 
Administration and Innovation Foundation of Graduate Students of Guizhou Normal University (no. 2012(11)).

\section{References}

[1] J. Shen, T. Tang, and L.-L. Wang, Spectral Methods: Algorithms, Analysis and Applications, vol. 41 of Springer Series in Computational Mathematics, Springer, Heidelberg, Germany, 2011.

[2] C. Canuto, M. Y. Hussaini, A. Quarteroni, and T. A. Zang, Spectral Methods: Fundamentals in Single Domains, Scientific Computation, Springer, Heidelberg, Germany, 2006.

[3] M. G. Armentano, C. Padra, R. Rodríguez, and M. Scheble, "An $h p$ finite element adaptive scheme to solve the laplace model for fluid-solid vibrations," Computer Methods in Applied Mechanics and Engineering, vol. 200, no. 1-4, pp. 178-188, 2011.

[4] H. Bi and Y. Yang, "Multiscale discretization scheme based on the Rayleigh quotient iterative method for the Steklov eigenvalue problem," Mathematical Problems in Engineering, vol. 2012, Article ID 487207, 18 pages, 2012.

[5] C. Carstensen, J. Gedicke, V. Mehrmann, and A. Miedlar, "An adaptive homotopy approach for non-selfadjoint eigenvalue problems," Numerische Mathematik, vol. 119, no. 3, pp. 557-583, 2011.

[6] X. Dai and A. Zhou, "Three-scale finite element discretizations for quantum eigenvalue problems," SIAM Journal on Numerical Analysis, vol. 46, no. 1, pp. 295-324, 2007.

[7] S. Giani and I. G. Graham, "A convergent adaptive method for elliptic eigenvalue problems," SIAM Journal on Numerical Analysis, vol. 47, no. 2, pp. 1067-1091, 2009.

[8] K. Kolman, "A two-level method for nonsymmetric eigenvalue problems," Acta Mathematicae Applicatae Sinica, vol. 21, no. 1, pp. 1-12, 2005.

[9] A. Naga and Z. Zhang, "Function value recovery and its application in eigenvalue problems," SIAM Journal on Numerical Analysis, vol. 50, no. 1, pp. 272-286, 2012.

[10] Y. Yang and X. Fan, "Generalized rayleigh quotient and finite element two-grid discretization schemes," Science in China A, vol. 52, no. 9, pp. 1955-1972, 2009.

[11] Y. Yang and H. Bi, "Two-grid finite element discretization schemes based on shifted-inverse power method for elliptic eigenvalue problems," SIAM Journal on Numerical Analysis, vol. 49, no. 4, pp. 1602-1624, 2011.

[12] Y. Yang, Y. Zhang, and H. Bi, "Multigrid discretization and iterative algorithm for mixed variational formulation of the eigenvalue problem of electric field," Abstract and Applied Analysis, vol. 2012, Article ID 190768, 25 pages, 2012.

[13] Y. Yang, W. Jiang, Y. Zhang, W. Wang, and H. Bi, "A twoscale discretization scheme for mixed variational formulation of eigenvalue problems," Abstract and Applied Analysis, vol. 2012, Article ID 812914, 29 pages, 2012.

[14] Y. Zhang, Y. Yang, and J. Liu, "Highly efficient calculation schemes of finite-element filter approach for the eigenvalue problem of electric field," Mathematical Problems in Engineering, vol. 2012, Article ID 529498, 21 pages, 2012.

[15] V. Heuveline and R. Rannacher, "A posteriori error control for finite element approximations of elliptic eigenvalue problems," Advances in Computational Mathematics, vol. 15, no. 1-4, pp. 107-138, 2001.

[16] Y. Yang and W. Jiang, "Upper spectral bounds and a posteriori error analysis of several mixed finite element approximations for the Stokes eigenvalue problem," Science China Mathematics, vol. 56, no. 6, pp. 1313-1330, 2013.

[17] I. Babuška and J. Osborn, "Eigenvalue problems," in Handbook of Numerical Analysis: Finite Element Methods (Part 1), P. G. Ciarlet and J. L. Lions, Eds., vol. 2, pp. 641-787, Elsevier Science North Holland, Amsterdam, The Netherlands, 1991.

[18] F. Chatelin, Spectral approximation of linear operators, Computer Science and Applied Mathematics, Academic Press, New York, NY, USA, 1983.

[19] Y. Yang, Finite Element Methods For Eigenvalue Problems, Science Press, Beijing, China, 2012, (Chinese).

[20] J. M. Melenk and B. I. Wohlmuth, "On residual-based a posteriori error estimation in $h p$-FEM," Advances in Computational Mathematics, vol. 15, no. 1-4, pp. 311-331, 2001.

[21] J. M. Melenk, " $h p$-interpolation of nonsmooth functions and an application to $h p$-a posteriori error estimation," SIAM Journal on Numerical Analysis, vol. 43, no. 1, pp. 127-155, 2005.

[22] P. Clément, "Approximation by finite element functions using local regularization," RAIRO Analyse Numérique, vol. 9, no. 2, pp. 77-84, 1975.

[23] L. R. Scott and S. Zhang, "Finite element interpolation of nonsmooth functions satisfying boundary conditions," Mathematics of Computation, vol. 54, no. 190, pp. 483-493, 1990.

[24] I. Babuška and M. R. Dorr, "Error estimates for the combined $h$ and $p$ versions of the finite element method," Numerische Mathematik, vol. 37, no. 2, pp. 257-277, 1981.

[25] J. Gedicke and C. Carstensen, A Posteriori Error Estimators For Non-Symmetric Eigenvalue Problems, DFG Research Center Matheon, Berlin, Germany, 2009. 


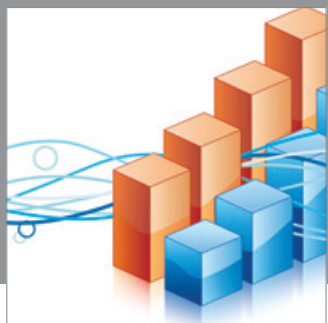

Advances in

Operations Research

mansans

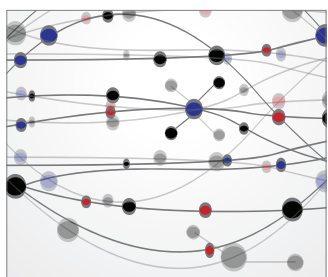

The Scientific World Journal
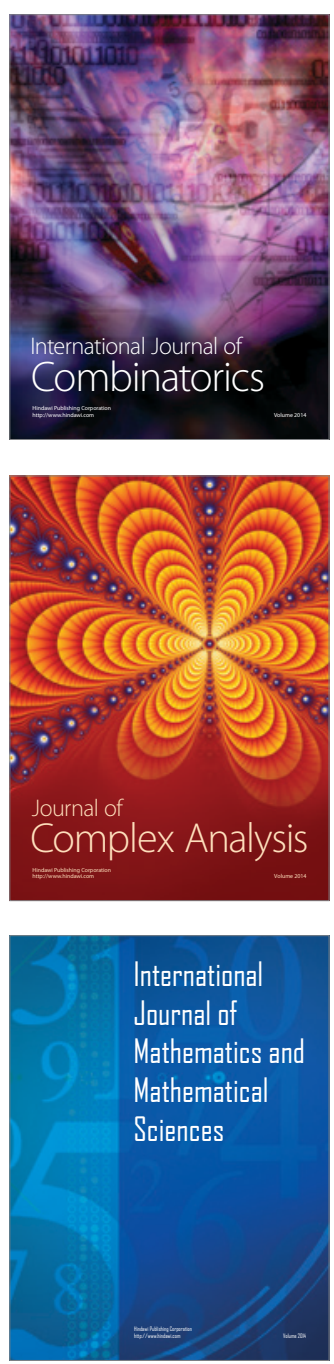
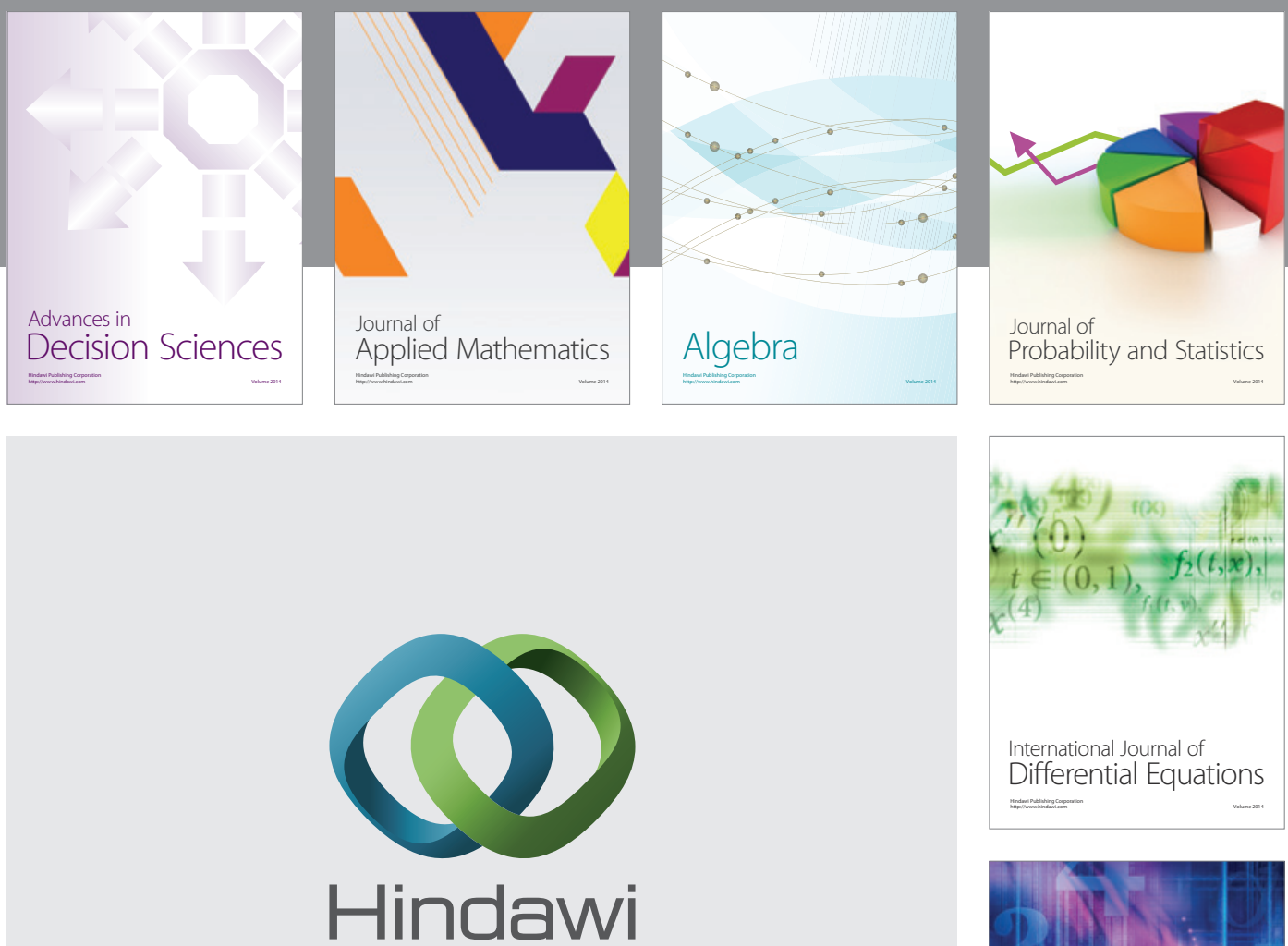

Submit your manuscripts at http://www.hindawi.com
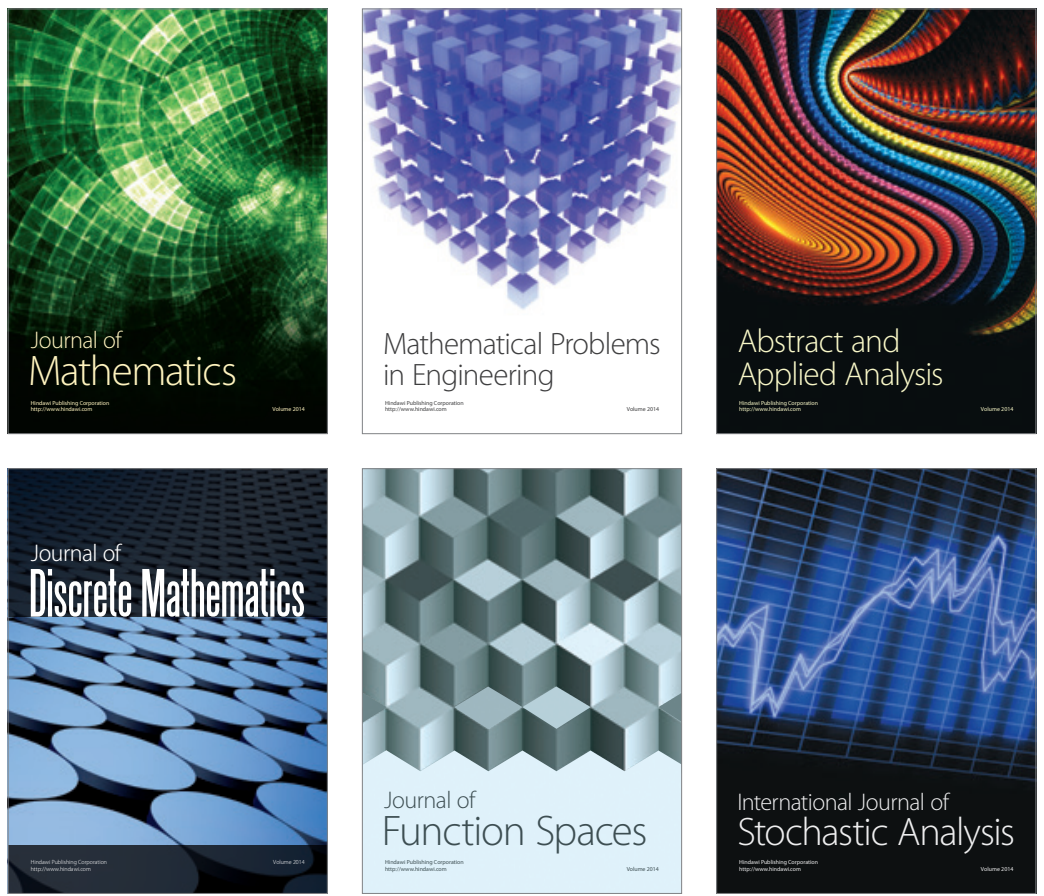

Journal of

Function Spaces

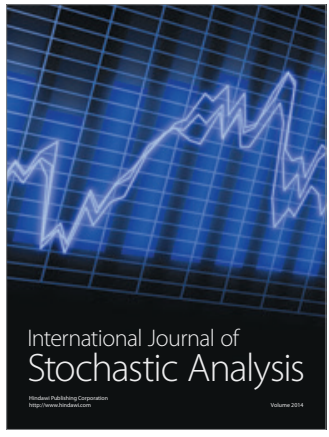

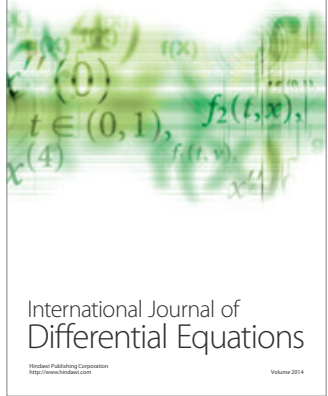
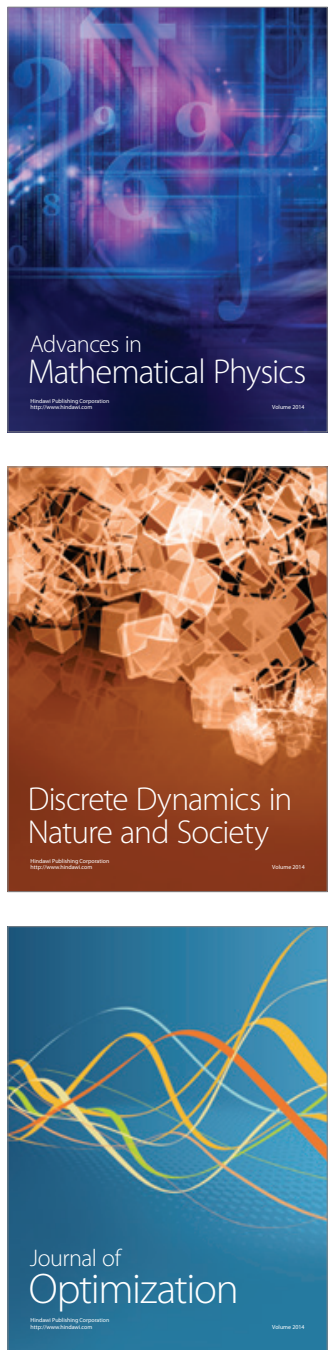